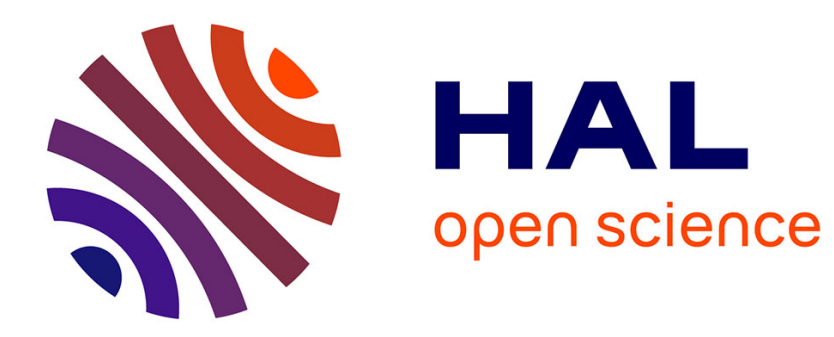

\title{
Synthèse et mesure des fréquences optiques
}

J.J. Jimenez

\section{To cite this version:}

J.J. Jimenez. Synthèse et mesure des fréquences optiques. Revue de Physique Appliquée, 1979, 14

(2), pp.353-367. 10.1051/rphysap:01979001402035300 . jpa-00244604

\section{HAL Id: jpa-00244604 https://hal.science/jpa-00244604}

Submitted on 1 Jan 1979

HAL is a multi-disciplinary open access archive for the deposit and dissemination of scientific research documents, whether they are published or not. The documents may come from teaching and research institutions in France or abroad, or from public or private research centers.
L'archive ouverte pluridisciplinaire HAL, est destinée au dépôt et à la diffusion de documents scientifiques de niveau recherche, publiés ou non, émanant des établissements d'enseignement et de recherche français ou étrangers, des laboratoires publics ou privés. 


\title{
Synthèse et mesure des fréquences optiques
}

\author{
J. J. Jimenez $(*)$ \\ Laboratoire Primaire du Temps et des Fréquences (LPTF). Observatoire de Paris et Bureau National de Métrologie \\ 61, avenue de l'Observatoire, 75014 Paris, France.
}

(Reçu le 30 juin 1978, révisé le 29 novembre 1978, accepté le 1er décembre 1978)

\begin{abstract}
Résumé. - Les lasers à gaz stabilisés en fréquence par absorption saturée sont actuellement acceptés comme étalons secondaires de fréquence et de longueur dans le domaine optique. Les caractéristiques de stabilité et reproductibilité en fréquence de ces différents lasers sont passées en revue.

La synthèse de fréquences, par mélange dans un dispositif non linéaire à large bande passante de plusieurs faisceaux lasers, permet la mesure de fréquences comprises entre les micro-ondes et l'infrarouge proche. La synthèse à partir de deux lasers à gaz carbonique nous a permis de mesurer la fréquence du laser He- $\mathrm{Ne} a ̀ ~ 88 \mathrm{THz}(3,39 \mu \mathrm{m})$. La plage de mesure peut être élargie en utilisant les différents isotopes du $\mathrm{CO}_{2}$.

Plusieurs types de dispositifs non linéaires sont capables de réaliser le mélange des fréquences optiques. Une étude de leurs caractéristiques comme détecteur et mélangeur montre les domaines respectifs possibles de fonctionnement. Plusieurs d'entre eux sont susceptibles de travailler à des fréquences infrarouges, et la possibilité d'étendre leur fonctionnement jusqu'au visible est signalée.

L'utilisation de nouveaux dispositifs, tels que l'oscillateur à cavité supraconductrice et le laser à alcool méthylique (qui possèdent des raies d'émission très étroites), dans un nouveau schéma de raccordement entre les fréquences radioélectriques et optiques, permet d'envisager la mesure de la fréquence du laser à méthane à $3,39 \mu \mathrm{m}$ avec une précision de l'ordre de $10^{-12}$.
\end{abstract}

\begin{abstract}
Saturated-absorption stabilized gas lasers provide, in the optical region, accepted secondary frequency and length standards. Frequency stability and reproducibility of these lasers are reviewed.

Using a broadband nonlinear device as a mixer, frequency synthesis of different lasers extend the frequency measurement range from microwaves to the near infrared. Using two carbon dioxide lasers, we have been able to measure the He-Ne laser frequency at $88 \mathrm{THz}(3.39 \mu \mathrm{m})$. The frequency measurement range may be increased using different $\mathrm{CO}_{2}$ isotopes.

Several nonlinear devices are able to mix optical frequencies from the submillimetric to the infrared wavelength. We review their characteristics as detectors and mixers, and we point out the possibility to extend it to the visible range.

The use of new devices, the cavity stabilized superconducting oscillator and the methyl alcohol laser, with very narrow linewidths, inserted in a new laser frequency synthesis chain, should permit measurements of the methane laser frequency at $3.39 \mu \mathrm{m}$ to parts in $10^{12}$.
\end{abstract}

1. Introduction. - Les lasers actuellement utilisés en métrologie et spectroscopie sont caractérisés par une grande pureté spectrale, une très haute stabilité et une excellente reproductibilité, qui peuvent atteindre des valeurs comprises entre $10^{-10}$ et $10^{-14}$ [1-3], [90]. On peut alors envisager de mesurer leur fréquence avec une très grande précision.

Actuellement, les techniques de mesure des fréquences optiques par raccordement à l'étalon de fréquence à césium permettent d'obtenir une précision de quelques $10^{-10}$ jusqu'à $88 \mathrm{THz}\left({ }^{* *}\right)$ et de l'ordre de

$\left({ }^{*}\right)$ Adresse actuelle : Institut d'Electronique Fondamentale (Laboratoire associé au C.N.R.S.), Université Paris XI, Bât. 220, 91405 Orsay Cedex, France.

$\left({ }^{* *}\right) 1 \mathrm{THz}=10^{12} \mathrm{~Hz}$.
$10^{-7}$ jusqu'à $197 \mathrm{THz}$ [3], fréquence la plus élevée qui ait été mesurée.

Lé domaine où il est possible de réaliser des mesures précises de fréquence recouvre ainsi largement la zone de mesures précises de longueur d'onde $[4,5]$.

Nous analysons la méthode employée pour la synthèse et la mesure des fréquences laser, ainsi que ses limitations. Le raccordement des fréquences entre l'étalon de temps et les lasers infrarouges sera présenté et nous montrerons la possibilité d'améliorer la précision de la mesure des fréquences optiques en utilisant des dispositifs, oscillateurs et diodes, très performants.

2. La stabilisation de fréquence des lasers à gaz. La fréquence émise par un laser dépend étroitement de la longueur optique de la cavité. Toute variation 
de cette longueur due aux vibrations ou aux effets thermiques se traduit par une variation de la fréquence à l'intérieur de la courbe de gain du milieu amplificateur.

Pour contrôler la longueur optique de la cavité d'un laser monomode, il est possible d'asservir la fréquence du laser :

a) sur une référence dont la largeur de raie est suffisamment étroite, en pratique, une raie non élargie par effet Doppler, ou

b) sur un signal dont la fréquence est synthétisée à partir d'une source ultra-stable.

La première technique est utilisée pour la plupart des lasers (pour une étude détaillée de cette technique, nous renvoyons le lecteur à la référence [6] dans cette même revue) : on emploie, par exemple, la fluorescence saturée dans le dioxyde de carbone pour stabiliser toutes les raies d'émission du $\mathrm{CO}_{2}$ [7], l'absorption saturée dans le $\mathrm{SF}_{6}$ pour stabiliser certaines raies d'émission du $\mathrm{CO}_{2}[8,9,10]$, dans le méthane (laser $\mathrm{He}-\mathrm{Ne}$ à $3,39 \mu \mathrm{m}[6,11])$ ou dans l'iode (laser He-Ne à 632,8 nm $[6,12]$ et laser à $\mathrm{Ar}^{+}$à $514,5 \mathrm{~nm}$ [13]). La figure 1 montre une comparaison de la stabilité de fréquence de ces lasers, caractérisée par la racine carrée de la variance d'Allan, $\sigma_{y}(\tau)$. La stabilité des horloges à césium $[1,2]$ et du meilleur oscillateur à cavité supraconductrice (SCSO) [14] est également indiquée.

En comparant ces courbes, on peut constater que certains lasers sont, à court terme, plus stables que l'étalon actuel à césium. En ce qui concerne l'exactitude et la reproductibilité, l'horloge à césium leur est encore supérieure $\left(10^{-13}\right)$ suivie par le maser à hydrogène $\left(10^{-12}\right)$ [1-3]. De ce point de vue, les valeurs typiques pour les lasers se trouvent dans la gamme de $10^{-10}$ à $10^{-11}$ [1-3], mais une reproductibilité possible de $3 \times 10^{-14}$ a été estimée pour le laser télescopique $\mathrm{He}-\mathrm{Ne}\left(\mathrm{CH}_{4}\right)$ [11], dont la courbe de stabilité est indiquée sur la figure 1.

Contrairement aux masers ou lasers, l'oscillateur à cavité supraconductrice (SCSO) n'utilise pas une résonance atomique ou moléculaire, mais une résonance électromagnétique dans une cavité supraconductrice, dont le coefficient de surtension $Q$ se situe entre $10^{8}$ et $10^{11}$ [15]. La stabilité de fréquence d'un générateur étant directement liée au coefficient de surtension de l'élément résonnant qui lui est associé, le SCSO est, à l'heure actuelle, le seul générateur dont la stabilité à court terme est meilleure que $10^{-15}$ (Fig. 1) [14], ce qui correspond à des largeurs de raie de l'ordre de quelques $\mu \mathrm{Hz}$. Comme on le verra plus loin ( $(5)$, il sera intéressant d'utiliser un tel générateur comme élément de départ d'une chaîne de synthèse de fréquences optiques. Les fréquences de résonance du SCSO s'étendent actuellement dans la gamme des micro-ondes, de 3 à $10 \mathrm{GHz}$ [15-17], mais leur utilisation semble possible jusqu'à des fréquences proches de $100 \mathrm{GHz}$. De tels oscillateurs sont actuellement

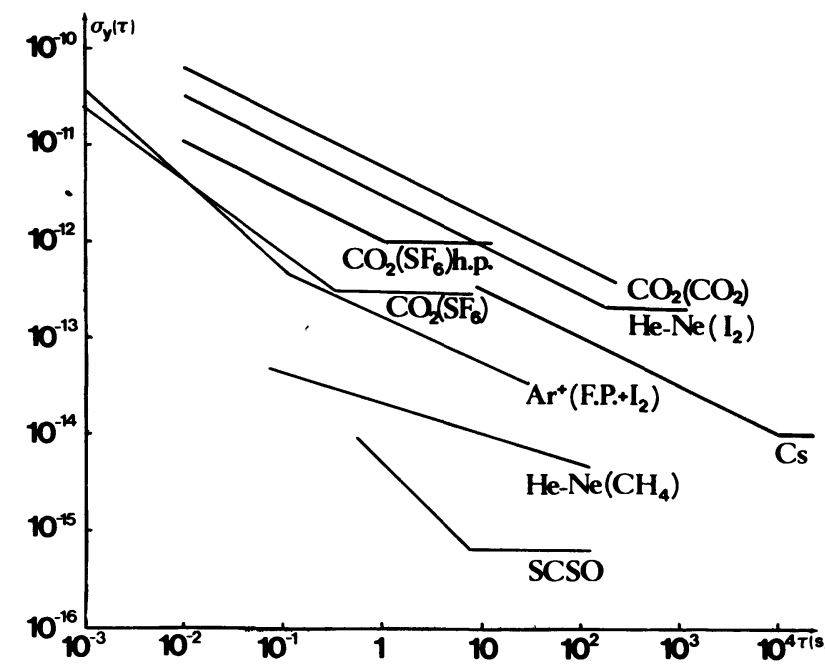

Fig. 1. - Comparaison de la stabilité de fréquence [1], caractérisée par la racine carrée de la variance d'Allan $\sigma_{y}(\tau)$, de différents lasers : $\mathrm{CO}_{2}\left(\mathrm{CO}_{2}\right)$, laser à $\mathrm{CO}_{2}$ à faible pression stabilisé sur la fluorescence saturée de $\mathrm{CO}_{2}[7] ; \mathrm{CO}_{2}\left(\mathrm{SF}_{6}\right)$, laser à $\mathrm{CO}_{2}$ à faible pression stabilisé sur l'absorption saturée de $\mathrm{SF}_{6}[9]: \mathrm{CO}_{2}\left(\mathrm{SF}_{6}\right)$ h.p., laser à $\mathrm{CO}_{2}$ à haute pression (guide d'onde) stabilisé sur l'absorption saturée de $\mathrm{SF}_{6}[10] ; \mathrm{He}-\mathrm{Ne}\left(\mathrm{I}_{2}\right)$, laser à He-Ne stabilisé sur l'absorption saturée de l'iode, avec cuve chaude [6, 12]: $\mathrm{He}-\mathrm{Ne}\left(\mathrm{CH}_{4}\right)$, laser à $\mathrm{He}-\mathrm{Ne}$ à télescope, stabilisé sur l'absorption saturée du méthane [11]; $\mathrm{Ar}^{+}\left(\mathrm{F} . \mathrm{P} .+\mathrm{I}_{2}\right)$, laser à argon ionisé stabilisé par une cavité Fabry-Pérot et sur l'absorption saturée de l'iode [13] : Cs, horloge à césium de laboratoire [1]; SCSO, meilleur oscillateur à cavité supraconductrice [14].

[Compared frequency stability, square root of the Allan variance $\sigma_{y}(\tau)$, of different lasers : $\mathrm{CO}_{2}\left(\mathrm{CO}_{2}\right)$, low pressure $\mathrm{CO}_{2}$ laser stabilized by $\mathrm{CO}_{2}$ saturated fluorescence [7] : $\mathrm{CO}_{2}\left(\mathrm{SF}_{6}\right)$, low pressure $\mathrm{CO}_{2}$ laser stabilized by $\mathrm{SF}_{6}$ saturated absorption [9]: $\mathrm{CO}_{2}\left(\mathrm{SF}_{6}\right)$ h.p., high pressure $\mathrm{CO}_{2}$ laser (waveguide) stabilized by $\mathrm{SF}_{6}$ saturated absorption [10]: He- $\mathrm{Ne}\left(\mathrm{I}_{2}\right)$, He-Ne laser stabilized by the $\mathrm{I}_{2}$ saturated absorption (hot cell) $[6,12]: \mathrm{He}-\mathrm{Ne}\left(\mathrm{CH}_{4}\right)$, telescopic $\mathrm{He}-\mathrm{Ne}$ laser stabilized by the $\mathrm{CH}_{4}$ saturated absorption [11]; $\mathrm{Ar}^{+}$(F.P. $+\mathrm{I}_{2}$ ), argon laser stabilized by a Fabry-Perot cavity and the $\mathrm{I}_{2}$ saturated absorption [13]; Cs, laboratory cesium clock [1] ; SCSO, best superconducting cavity stabilized oscillator [14].]

en cours d'étude à Orsay à 30 et $60 \mathrm{GHz}$ [18], en utilisant comme référence une cavité Fabry-Pérot à miroirs et blindage supraconducteurs.

La deuxième technique (synthèse d'une fréquence à partir d'une source stable), semble être la plus prometteuse pour stabiliser les lasers dans l'infrarouge lointain (LIR), puisqu'on ne connaît pas actuellement de raie suffisamment étroite à la fréquence souhaitée. Les lasers LIR, pompés optiquement, peuvent être modulés très rapidement en utilisant l'effet Stark, et être ainsi asservis en phase sur une référence de fréquence $[19,20]$, comme nous le verrons plus loin $(\S 5)$.

3. Synthèse et mesure des fréquences laser. - La mesure des fréquences laser est réalisée en utilisant la méthode hétérodyne classique qui permet d'obtenir, à partir du laser à mesurer et d'une ou plusieurs sources de fréquence connue, une fréquence de battement plus basse, inférieure à $500 \mathrm{MHz}$, susceptible 
d'être mesurée directement grâce à un compteur électronique.

Plusieurs méthodes de mesure sont possibles, mais le système le plus couramment utilisé est représenté sur la figure 2 . Les fréquences connues d'un ou deux lasers sont mélangées dans un dispositif non linéaire, capable de produire la somme ou la différence de plusieurs fréquences ou le mélange entre le fondamental d'une fréquence et l'harmonique d'une fréquence inférieure. On ajoute dans certains cas le signal d'un générateur micro-onde stabilisé, pour obtenir le battement à une fréquence inférieure ou égale à $100 \mathrm{MHz}$. Ce signal de battement est amplifié, puis mesuré dans un analyseur de spectre avec une résolution de l'ordre de $10 \mathrm{~Hz}$.

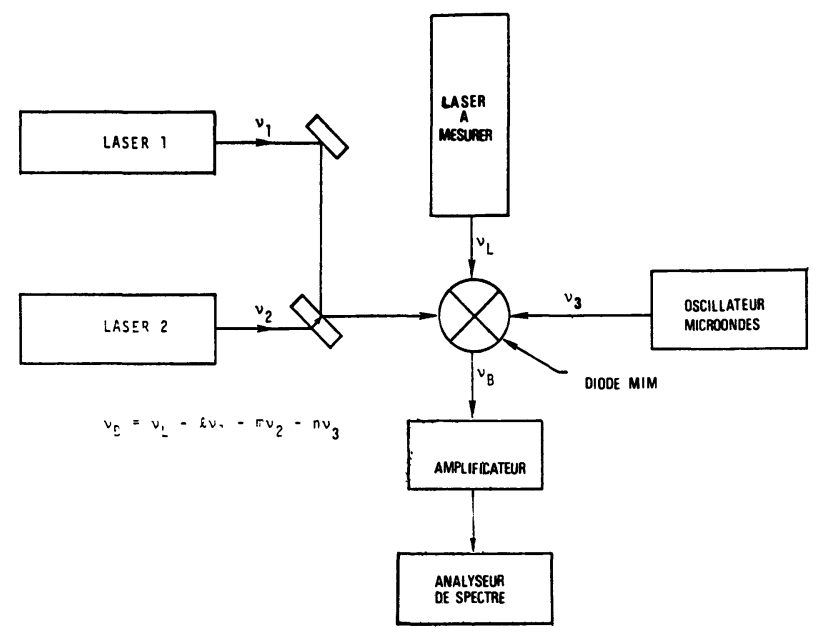

Fig. 2. - Schéma de principe de la mesure de la fréquence d'un laser par la méthode hétérodyne.

[Schematic diagram of a typical laser frequency synthesis experiment.]

D'une façon générale, la fréquence à mesurer $v_{\mathrm{L}}$ peut s'écrire :

$v_{\mathrm{L}}=l v_{1}+m v_{2}+n v_{3} \pm v_{\mathrm{B}}$

où $v_{1}$ et $v_{2}$ sont les fréquences de laser connues, $v_{3}$ la fréquence micro-onde et $v_{\mathrm{B}}$ la fréquence de battement. Les valeurs $l, m$ et $n$ sont des entiers positifs ou négatifs, en général petits, pour obtenir un ordre de mélange inférieur à dix.

L'utilisation de cette méthode a permis la mesure précise de la fréquence des lasers $\mathrm{H}_{2} \mathrm{O}$ et $\mathrm{D}_{2} \mathrm{O}$ avec une précision de $\pm 1 \mathrm{MHz}$ (limitation due à la difficulté de déterminer le centre de la raie du laser à mesurer [21]), ainsi que la mesure de 13 raies du laser $\mathrm{CH}_{3} \mathrm{OH}$, avec une précision de $\pm 0,5 \mathrm{MHz}$ [21]

3.1 MeSure de LA FRÉQUence DU LASER He-Ne $(3,39 \mu \mathrm{m})$. - En utilisant le même principe de mesure, nous avons réalisé dernièrement la mesure de la fréquence du laser à He-Ne à 3,39 $\mu \mathrm{m}$. Le laser de $8 \mathrm{~m}$ de long, a été décrit antérieurement [22, 23]. Pour cette expérience, il a été rempli avec un mélange de ${ }^{3} \mathrm{He}-{ }^{22} \mathrm{Ne}$, avec des pressions partielles de $400 \mathrm{~Pa}$ et 53,4 Pa, respectivement. Ce laser monomode fournit une puissance de $25 \mathrm{~mW}$ à $3,39 \mu \mathrm{m}$.

La fréquence a été mesurée en utilisant comme élément non linéaire une diode à pointe MIM (métalisolant-métal) réalisée au laboratoire $(\S 4.1 .2)$. Le mélange, d'ordre cinq, permet d'obtenir la valeur de la fréquence du laser à partir de l'expression (1) :

$$
\begin{gathered}
v_{\mathrm{He}-\mathrm{Ne}}=2 v_{12} \mathrm{C}^{16} \mathrm{O}_{2} \mathrm{P}_{\mathrm{II}}(18) \\
+v_{12 \mathrm{C}^{16} \mathrm{O}_{2} \mathrm{P}_{\mathrm{II}}(10)}+ \\
+v_{\text {micro-ondes }}+v_{\text {battement }} .
\end{gathered}
$$

La fréquence micro-onde obtenue à partir d'un klystron stabilisé, travaillant à $9,7 \mathrm{GHz}$, est mesurée grâce à un compteur électronique : les fréquences des raies $\mathrm{P}_{\mathrm{I}}(18)$ et $\mathrm{P}_{\mathrm{II}}(10)$ du ${ }^{12} \mathrm{C}^{16} \mathrm{O}_{2}$ sont connues à partir de mesures antérieures [24] et la fréquence de battement est mesurée à l'aide d'un analyseur de spectre. Les trois lasers $\left(\mathrm{He}-\mathrm{Ne}\right.$ et $\left.\mathrm{CO}_{2}\right)$ utilisés dans l'expérience ne sont pas asservis sur une résonance moléculaire (lasers libres). La stabilité des lasers à $\mathrm{CO}_{2}$ [23] est dans ces conditions $\sigma_{y}(\tau)=2,2 \times 10^{-11}$ pour $\tau<0,1 \mathrm{~s}$, et $\sigma_{y}(\tau)=1,8 \times 10^{-10} \tau$ pour $0,1 \mathrm{~s}<\tau<10 \mathrm{~s}$.

La diode MIM (\$4.1.2) est constituée par une base en nickel poli optiquement et oxydé naturellement en contact avec un whisker en tungstène terminé par une pointe de $50 \mathrm{~nm}$ de rayon [23]. Elle est obtenue par électrolyse dans une solution $\mathrm{NaOH}(2 \mathrm{~N})$, à partir d'un fil de $25 \mu \mathrm{m}$ de diamètre. A une distance $L=120 \mu \mathrm{m}$ de la pointe, le fil est coudé à $90^{\circ}$, ce qui permet de constituer une antenne [25], excitée par le rayonnement incident. Pour chaque rayonnement, le rendement est maximum pour un angle d'incidence particúlier ( $\S 4.1)$. Ainsi, pour un rapport $L / \lambda=35$ à $\lambda=3,39 \mu \mathrm{m}$, l'angle optimum est $\theta=11^{\circ}$, et il serait de $\theta=14^{\circ}$ pour $\lambda=10 \mu \mathrm{m}$ [25]. Les faisceaux lasers, polarisés linéairement, sont focalisés sur l'antenne avec des lentilles en silice de $25 \mathrm{~mm}$ de distance focale pour le rayonnement à $3,39 \mu \mathrm{m}$ d'une part, et en $\mathrm{KCl}$ de $50 \mathrm{~mm}$ de distance focale pour le rayonnement à $10 \mu \mathrm{m}$ d'autre part. Les deux faisceaux $\mathrm{CO}_{2}$ sont préalablement superposés grâce à une lame semiréfléchissante. Les valeurs mesurées pour l'impédance de la diode sont comprises entre 200 et $600 \Omega$. La diode est stable pendant plusieurs heures et peut être réutilisée pour la même expérience pendant deux semaines.

Le signal de battement est amplifié dans un amplificateur large bande $(0,1-1200 \mathrm{MHz})$ et mesuré à l'aide d'un analyseur de spectres $110 \mathrm{MHz}$ avec une résolution de $10 \mathrm{~Hz}$.

La figure 3 montre le spectre du signal de battement à $43 \mathrm{MHz}$ pour un temps de balayage de $5 \mathrm{~ms}$ par division. Le meilleur rapport signal/bruit obtenu ne dépasse pas $15 \mathrm{~dB}$, à cause de la relativement faible puissance du laser $\mathrm{He}-\mathrm{Ne}$. 


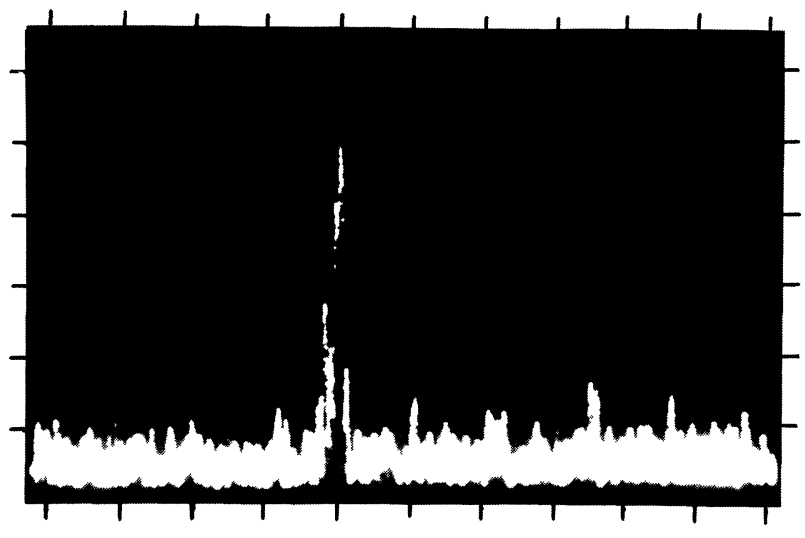

Fig. 3. - Spectre du signal de battement à $43 \mathrm{MHz}$ obtenu à partir de la synthèse de fréquence du laser He-Ne à $3,39 \mu \mathrm{m}$. Echelles horizontale $20 \mathrm{kHz}$ par division, verticale linéaire $2 \mu \mathrm{V}$ par division ; largeur de bande RF, $3 \mathrm{kHz}$; largeur de bande vidéo, $10 \mathrm{kHz}$; temps de balayage $5 \mathrm{~ms}$ par division.

[Beat note at $43 \mathrm{MHz}$ between the He-Ne laser at $3.39 \mu \mathrm{m}$ and the synthetized frequency. Horizontal scale, $20 \mathrm{kHz} /$ division; linear vertical scale, $2 \mu \mathrm{V} /$ division; $\mathrm{RF}$ bandwidth, $3 \mathrm{kHz}$; video bandwidth, $10 \mathrm{kHz}$; sweep time, $5 \mathrm{~ms} /$ division.]

La largeur de raie à mi-hauteur du laser est de l'ordre de $6 \mathrm{kHz}$, valeur 80 fois plus faible que celle observée dans des expériences antérieures similaires aux nôtres [26, 27].

La valeur de la fréquence obtenue à partir de notre mesure, avec les trois lasers libres, est égale à

$v_{\mathrm{He}-\mathrm{Ne}}=88376208 \pm 29 \mathrm{MHz}$

elle est comparable avec le résultat des premières mesures réalisées à cette fréquence [28] qui fournissent la valeur:

$v_{\mathrm{He}-\mathrm{Ne}}=88376200 \pm 50 \mathrm{MHz}$.

Ce résultat démontre bien l'intérêt de la méthode de synthèse de fréquences laser utilisant deux lasers $\mathrm{CO}_{2}$.

3.2 SYNTHÈSE DES FRÉQUENCES OPTIQUES PAR BATTEMENT DE DEUX LASERS $\mathrm{CO}_{2}$. - L'utilisation de lasers $\mathrm{CO}_{2}$ stabilisés sur la fluorescence saturée du $\mathrm{CO}_{2}$ permet, sans changer le gaz ni ses conditions de pression, d'employer ce laser comme un étalon secondaire de fréquence dans une très large gamme. Ainsi, la seule utilisation du ${ }^{12} \mathrm{C}^{16} \mathrm{O}_{2}$ permet d'obtenir plus de 100 fréquences entre 27 et $33 \mathrm{THz}$, reproductibles à mieux que $2 \times 10^{-10}$ près et très stables (mieux que $10^{-11}$ ) avec une puissance suffisante, de l'ordre du watt, pour la génération d'harmoniques.

En utilisant deux lasers à ${ }^{12} \mathrm{C}^{16} \mathrm{O}_{2}$ pour la synthèse de fréquences, on peut obtenir approximativement 7000 fréquences dans la bande $25 \mathrm{GHz}$ à $5,8 \mathrm{THz}$, avec une incertitude inférieure à $30 \mathrm{kHz}$, par différence entre les 60 raies existantes dans l'intervalle 27 à $33 \mathrm{THz}(9,1$ à $11 \mu \mathrm{m})$. En fait, on peut obtenir une série de fréquences discrètes comprises entre $25 \mathrm{GHz}$ et $100 \mathrm{THz}$, avec des ordres de mélange des fréquences $\mathrm{du}{ }^{12} \mathrm{C}^{16} \mathrm{O}_{2}$ inférieurs à 10 .

Pour cette raison, nous avons entrepris la réalisation de deux cellules externes de stabilisation par fluorescence saturée, fonctionnant à la température ambiante [23, 24]. La figure 4 montre le signal de fluorescence à $4,3 \mu \mathrm{m}$ entre le niveau $\left(00^{\circ} 1\right)$ et le niveau $\left(00^{\circ} 0\right)$ $\mathrm{du}^{12} \mathrm{C}^{16} \mathrm{O}_{2}$ contenu dans la cellule à une pression de $5,3 \mathrm{~Pa}$, lorsqu'il est excité par une puissance d'environ $1 \mathrm{~W}$ de la raie $\mathrm{P}_{\mathrm{II}}(10)$ du laser à $\mathrm{CO}_{2}$. Cette cellule est constituée par un tube en pyrex de $150 \mathrm{~mm}$ de longueur, doré intérieurement. L'émission à $4,3 \mu \mathrm{m}$ est détectée par une cellule photovoltaïque SbIn refroidie, de détectivité $D^{*}=2,3 \times 10^{10} \mathrm{~cm} \mathrm{~W}^{-1} \mathrm{~Hz}^{1 / 2}$. Un filtre de $1 \mu \mathrm{m}$ de largeur de bande centré à $4,3 \mu \mathrm{m}$ réduit la bande passante de bruit. Le rapport signal/bruit mesuré $(S / B(\Delta f=1 \mathrm{~Hz})=400)$ est suffisant pour asservir la fréquence du laser sur le pic de fluorescence. Il peut être augmenté en utilisant des détecteurs de grande surface et de détectivité élevée, supérieure à $10^{11}$, associés à un réflecteur de rayonnement porté à la température de l'azote liquide [7]. Ceci permet d'obtenir une stabilité de fréquence donnée par $\sigma_{y}(\tau)=6 \times 10^{-12} \tau^{-1 / 2}$ pour $10^{-2} \mathrm{~s}<\tau \leqslant 10^{2} \mathrm{~s}$, comme le montre la figure 1 [8].

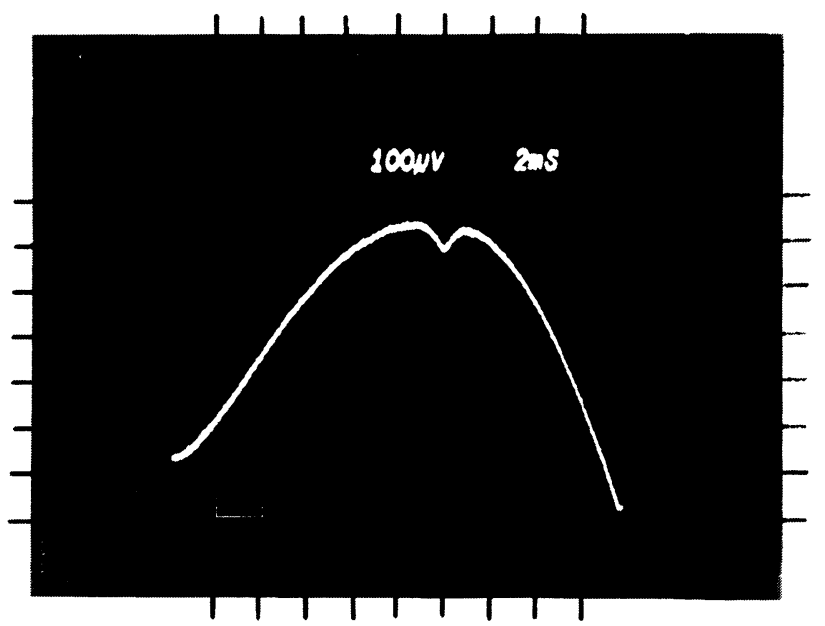

Fig. 4. - Signal de fluorescence de la cellule d'absorption externe à $\mathrm{CO}_{2}$ excitée par la raie $\mathrm{R}_{\mathrm{II}}(10)$. Pression dans la cellule 5,3 $\mathrm{Pa}$; bande passante du détecteur et de l'amplificateur, $10 \mathrm{kHz}$; puissance du laser, environ $1 \mathrm{~W}$.

[Fluorescence signal from the external $\mathrm{CO}_{2}$ absorption cell at $\mathbf{R}_{\mathrm{II}}(10)$. Cell pressure $5.3 \mathrm{~Pa}$; detector and amplifier bandwidth, $10 \mathrm{kHz}$; laser power, about $1 \mathrm{~W}$.]

D'autre part, la plage de fréquences utiles du laser à $\mathrm{CO}_{2}$ peut être élargie. $\mathrm{Le}^{12} \mathrm{C}^{16} \mathrm{O}_{2}$ couvre la gamme 9,1 à $9,9 \mu \mathrm{m}$ et 10,1 à $11 \mu \mathrm{m}$. En utilisant les transitions fournies par d'autres isotopes du $\mathrm{CO}_{2}$ [29-31] $\left({ }^{12} \mathrm{C}^{18} \mathrm{O}_{2},{ }^{13} \mathrm{C}^{16} \mathrm{O}_{2},{ }^{13} \mathrm{C}^{18} \mathrm{O}_{2}\right.$ et $\left.{ }^{14} \mathrm{C}^{16} \mathrm{O}_{2}\right)$, la plage de fréquences utiles du laser à gaz carbonique couvre alors toute la gamme de 9 à $12,4 \mu \mathrm{m}$ qui peut encore être étendue jusqu'à $12,5 \mu \mathrm{m}(24$ à $33,5 \mathrm{THz})$ en 
employant les "bandes chaudes» du ${ }^{12} \mathrm{C}^{16} \mathrm{O}_{2}$ et ${ }^{14} \mathrm{C}^{16} \mathrm{O}_{2}[30,31]$. La figure 5 montre le domaine des fréquences utiles couvert avec le laser à $\mathrm{CO}_{2}$ en utilisant quatre isotopes. Les isotopes plus complexes ${ }^{16} \mathrm{O}^{13} \mathrm{C}^{18} \mathrm{O}$ et ${ }^{16} \mathrm{O}^{12} \mathrm{C}^{18} \mathrm{O}$ ne sont pas considérés ici [30]. En fait, la seule utilisation du ${ }^{12} \mathrm{C}^{16} \mathrm{O}_{2}$ et du ${ }^{14} \mathrm{C}^{16} \mathrm{O}_{2}$, avec ses bandes chaudes pourrait permettre de couvrir presque complètement la bande 24 à $33 \mathrm{THz}$, mais le fait que les puissances de sortie les plus importantes du laser à $\mathrm{CO}_{2}$ se trouvent au centre des bandes $\mathrm{P}$ et $\mathrm{R}$, nous incite à employer $\mathrm{le}^{13} \mathrm{C}^{16} \mathrm{O}_{2}$. Cette idée sera mise en œuvre dans la nouvelle chaîne de synthèse de fréquences laser $(\S 5)$, où la raie $P_{n}(28)$ à $10,1 \mu \mathrm{m}$ du ${ }^{13} \mathrm{C}^{16} \mathrm{O}_{2}$ sera utilisée au lieu de la raie $\mathrm{R}_{\mathrm{II}}(10)$ à $9,3 \mu \mathrm{m} \mathrm{du}{ }^{12} \mathrm{C}^{16} \mathrm{O}_{2}$, qui est d'usage courant dans la chaîne de synthèse de fréquences laser classique $[2-5,23]$.
La figure 6 montre un battement obtenu dans une diode MIM entre deux lasers $\mathrm{CO}_{2}$ non stabilisés fonctionnant sur les raies $\mathrm{P}_{\mathrm{I}}(34) \mathrm{du}{ }^{12} \mathrm{C}^{16} \mathrm{O}_{2}$ pour l'un et $\mathrm{R}_{\mathrm{T}}(24) \mathrm{du}{ }^{13} \mathrm{C}^{16} \mathrm{O}_{2}$ pour l'autre (laser à enceinte scellée). La différence de fréquence mesurée dans cette expérience préliminaire est d'environ $9225 \mathrm{MHz}$, valeur en assez bon accord avec celle rapportée par Freed et al. [29] (égal à $9230,3 \mathrm{MHz}$ ).

4. Les diodes. - La mesure de fréquences laser ne serait pas possible sans le dispositif non linéaire capable de produire des harmoniques et d'effectuer le mélange de plusieurs radiations optiques $[4,32,33]$. Ce dispositif doit posséder une bande passante de plusieurs $\mathrm{GHz}$, ce qui exclut les détecteurs thermiques ou photoniques, dont la bande passante est en général inférieure à $100 \mathrm{MHz}$. Seule la photodiode en $\mathrm{Cd}-\mathrm{Hg}$ -

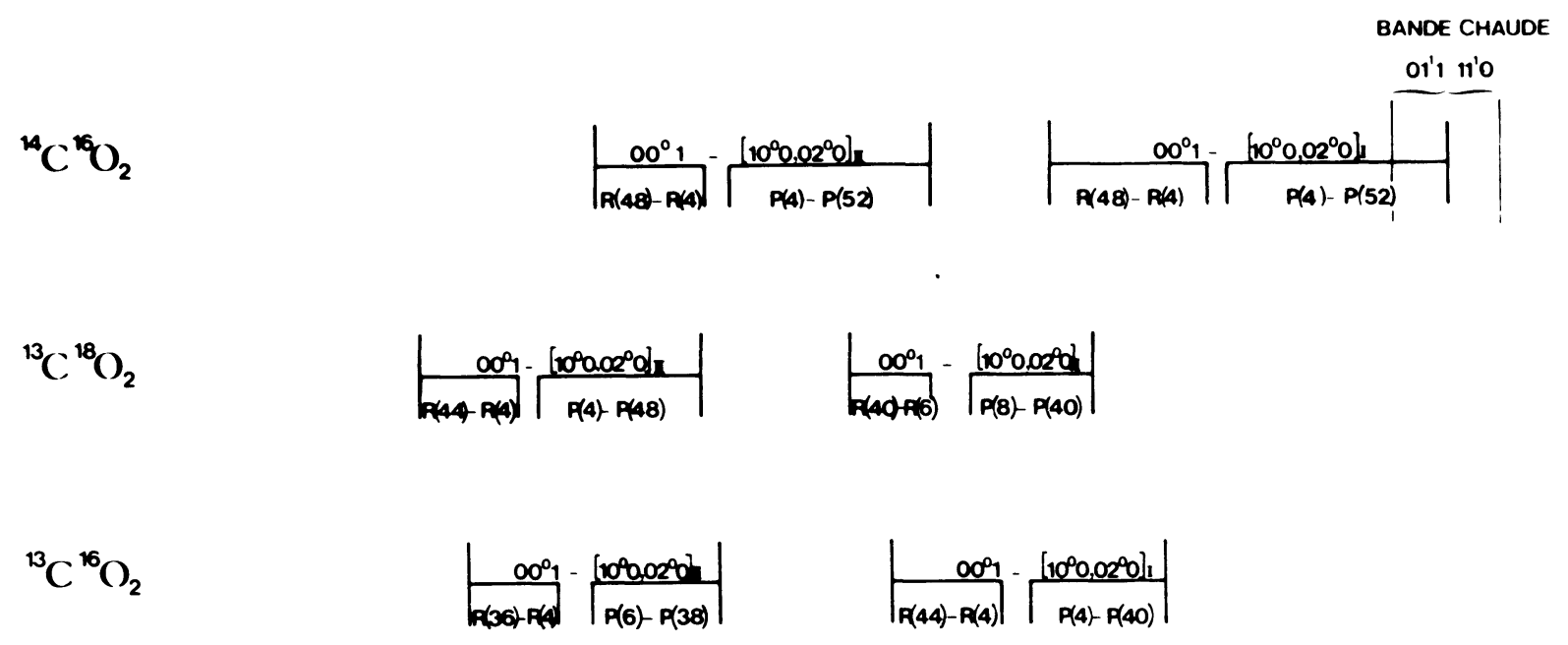

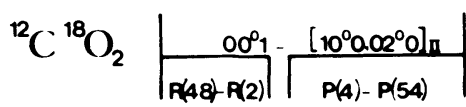

$\left|\frac{\infty 0^{\circ} 1-}{\mid+(38)-1(6)}-\frac{\left[10^{\circ} 0.0207\right]}{P(8)-P(40)}\right|$

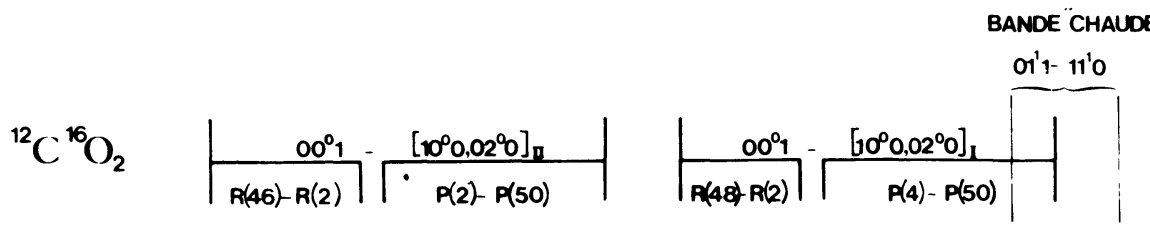

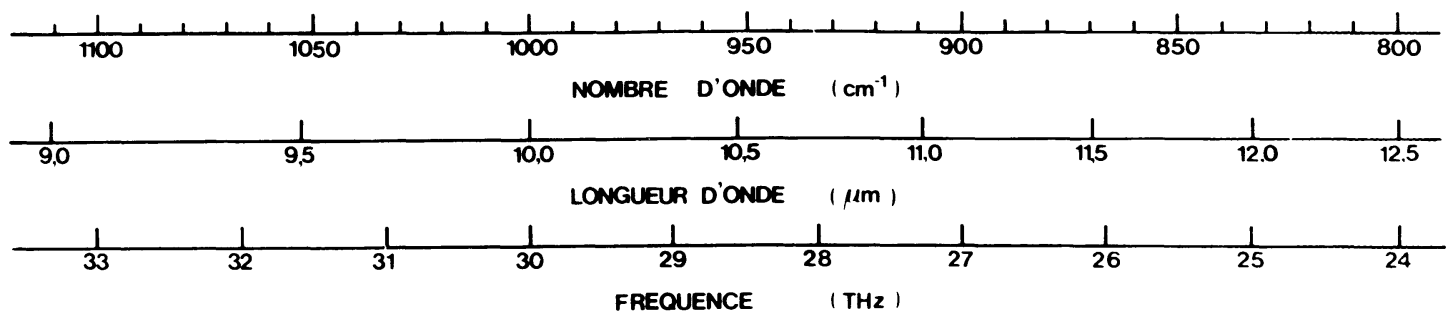

Fig. 5. - Comparaison des plages de fréquence et longueur d'onde des lasers à gaz carbonique et de quatre de ses isotopes.

[Comparison of the frequency and wavelength domains of four rare $\mathrm{CO}_{2}$ isotope lasers with ${ }^{12} \mathrm{C}^{16} \mathrm{O}_{2}$.] 


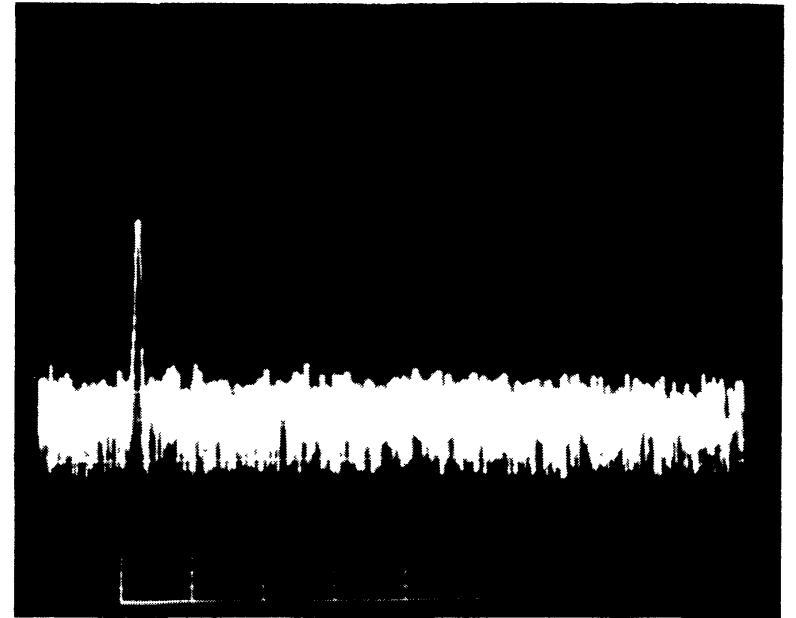

Fig. 6. - Spectre du signal de battement à $25 \mathrm{MHz}$ entre les raies $\mathrm{P}_{\mathrm{I}}(34) \mathrm{du}{ }^{12} \mathrm{C}^{16} \mathrm{O}_{2}$ et $\mathrm{R}_{\mathrm{I}}(24) \mathrm{du}{ }^{13} \mathrm{C}^{16} \mathrm{O}_{2}$. Echelles : horizontale, $50 \mathrm{kHz}$ par division; verticale, $10 \mathrm{~dB}$ par division; largeur de bande $\mathrm{RF}, 3 \mathrm{kHz}$, largeur de bande vidéo, $10 \mathrm{kHz}$; temps de balayage $20 \mathrm{~ms}$ par division.

[Beat note at $25 \mathrm{MHz}$ between the ${ }^{12} \mathrm{C}^{16} \mathrm{O}_{2} \mathrm{P}_{\mathrm{I}}(34)$ and ${ }^{13} \mathrm{C}^{16} \mathrm{O}_{2}$ $\mathbf{R}_{\mathrm{I}}(24)$ lines. Horizontal scale $50 \mathrm{kHz} /$ division ; vertical scale $10 \mathrm{~dB} /$ division: $\mathrm{RF}$ bandwidth, $3 \mathrm{kHz}$; video bandwidth, $10 \mathrm{kHz}$; sweep time, $20 \mathrm{~ms} /$ division.]

Te refroidie à l'azote, et seulement pour des échantillons spécialement sélectionnés, possède des largeurs de bande de l'ordre du $\mathrm{GHz}$, dans la bande des fréquences des lasers à $\mathrm{CO}_{2}$.

Les dispositifs de volume [32] (cristaux non linéaires) ont été également utilisés pour la synthèse de fréquences laser, mais, à cause des faibles rendements obtenus, il faut faire une adaptation de phase, ce qui limite leur utilisation au deuxième harmonique. Toutefois, et par manque d'autres types de dispositif, on envisage leur emploi pour la mesure des fréquences visibles, par doublement d'une fréquence infrarouge [34].

Les dispositifs couramment utilisés pour la synthèse de fréquences optiques sont en général les diodes à pointes ; des diodes à structure planar, en cours de développement, ont pu être utilisées également dans l'infrarouge.

Les techniques utilisées pour la réalisation des diodes travaillant dans le domaine optique (IR), extrapolent les techniques développées en microondes pour la réalisation des diodes mélangeuses. Trois articles récents $[4,32,33]$ ont étudié l'application de ces dispositifs à la mesure des fréquences optiques. Le tableau I en résume les principales caractéristiques.

4.1 Diodes a POINTES. EFFET D'ANTENNE DÛ AU " WHISKER ". - Nous pouvons distinguer dans cette catégorie trois types de diodes : métal-semiconducteur (MS), métal-isolant-métal (MIM) et supraconducteurisolant-supraconducteur (jonction Josephson).

Il est important de souligner l'effet de gain dû au whisker utilisé dans ces diodes. La portion de fil proche du contact se comporte comme une antenne à onde progressive [25], dont le lobe principal forme avec l'axe un angle $\theta$ donné par l'expression :

$\theta=\arccos \left(1-0,371 \frac{\lambda}{L}\right)$

où $\lambda$ est la longueur d'onde du rayonnement incident et $L$ une longueur effective d'antenne délimitée sur le whisker par une forte discontinuité (Fig. 7). L'intérêt du modèle est de pouvoir prédire avec une précision satisfaisante les directions des lobes et, de là, les directions d'incidence assurant un bon couplage (\$3.1). Des confrontations expérimentales en vraie grandeur à $337 \mu \mathrm{m}$ [25], 10,6 $\mu \mathrm{m}$ [35] et même $3,39 \mu \mathrm{m}$ [35] confirment le bien-fondé de cette hypothèse.

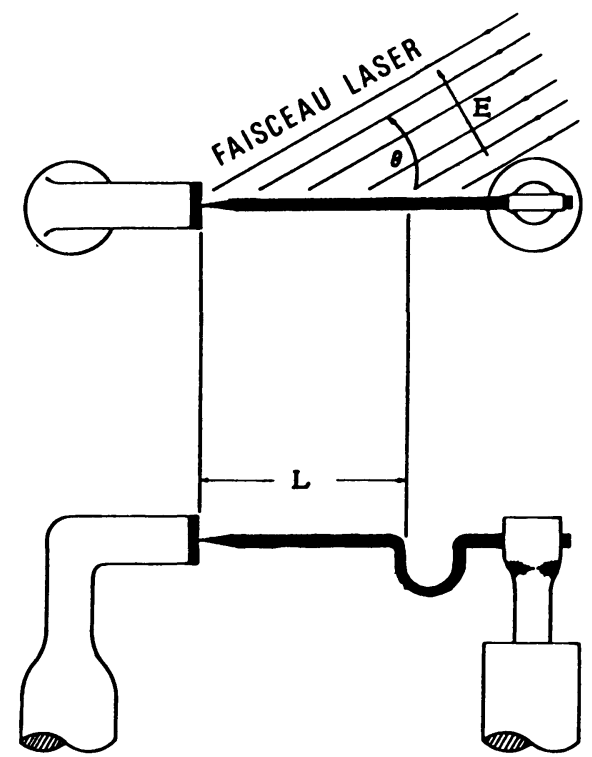

Fig. 7. - Couplage du rayonnement optique à l'antenne d'une diode à pointes (d'après réf. [25]).

[Coupling between the laser beam and the antenna whisker in a point contact diode (from [25])].

En utilisant une telle antenne optique pour coupler le rayonnement d'un laser $\mathrm{HCN}(337 \mu \mathrm{m})$ à une diode Schottky, on obtient une variation linéaire du gain de l'antenne de 10 à 100 pour des valeurs de $L / \lambda$ comprises entre 10 et 100 [36]. Ce même effet de gain a permis, pour la première fois, le couplage d'un rayonnement à $3,39 \mu \mathrm{m}$ à un détecteur photovoltaïque en SbIn [37].

Des études sont actuellement en cours $[38,39]$ pour optimiser le couplage entre le rayonnement et l'antenne. L'utilisation de réflecteurs placés à une distance du " whisker ", de l'ordre de la longueur d'onde du rayonnement laser, permet des gains supplémentaires de $12 \mathrm{~dB}$ dans le domaine submillimétrique [40].

4.1.1 La diode métal-semiconducteur. - La diode métal-semiconducteur est fondamentalement une jonction à barrière Schottky. La partie métallique peut 
constituer en même temps l'antenne qui couple le rayonnement laser à la jonction. La diode à pointe à semiconducteur (tungstène-silicium) fut la première utilisée dans le domaine optique [41]. Semblable à la diode utilisée dans les premiers récepteurs radio, elle a permis d'obtenir des ordres de multiplication élevés, mais sa fréquence limite est relativement basse, de l'ordre de quelques $\mathrm{THz}$ (Tableau I). L'utilisation de diodes tungstène-germanium [42] a rendu possible la détection de longueurs d'onde de 10,6 $\mu \mathrm{m}$ avec des

Tableau I. - Caractéristiques des détecteurs-mélangeurs dans le domaine optique. Certaines données proviennent de communications privées, mais la plupart ont été obtenues de publications récentes (voir texte). Pour un dispositif de type donné, les valeurs indiquées sur le tableau ne proviennent pas nécessairement de la même expérience.

\begin{tabular}{|c|c|c|c|c|c|c|c|c|}
\hline \multirow{3}{*}{ Détecteur } & \multicolumn{3}{|c|}{ Détection (non hétérodyne) } & \multicolumn{5}{|c|}{ Générateur d'harmoniques et mélangeur (hétérodyne) } \\
\hline & \multirow{2}{*}{$\begin{array}{l}\text { Longueur } \\
\text { d'onde } \\
\text { limite } \\
(\mu \mathrm{m})\end{array}$} & \multicolumn{2}{|c|}{$\begin{array}{l}\text { Bruit } N E P\left({ }^{a}\right) \\
\text { à la fréquence } v\end{array}$} & \multirow{2}{*}{$\begin{array}{c}\text { Fréquence } \\
\text { limite } v_{1}\left({ }^{(}\right) \text {, } \\
v_{2}\left({ }^{d}\right) \\
(\mathrm{THz})\end{array}$} & \multirow{2}{*}{$\begin{array}{l}\text { Ordre } \\
\text { de } \\
\text { multipli- } \\
\text { cation }\end{array}$} & \multirow{2}{*}{$\begin{array}{c}\text { Temps de } \\
\text { réponse } \\
-\left({ }^{e}\right) \\
\tau=1 / v_{1} \text { (fs) }\end{array}$} & \multicolumn{2}{|c|}{$\begin{array}{l}\text { Bruit } M D P\left({ }^{b}\right) \\
\text { à la fréquence } v\end{array}$} \\
\hline & & $N E P\left(\mathrm{~W} \mathrm{~Hz}^{-1 / 2}\right)$ & $v(\mathrm{THz})$ & & & & $M D P\left(\mathrm{~W} \mathrm{~Hz}^{-1}\right)$ & $v(\mathrm{THz})$ \\
\hline $\begin{array}{l}\text { 1.3 Supraconducteur- } \\
\text { isolant-supra- } \\
\text { conducteur } \\
\text { Jonction } \\
\text { Josephson (CP) } \\
\text { 2. Diodes à structure } \\
\text { planar } \\
\text { 2.1 Métal-semi- } \\
\text { conducteur (MS) } \\
\text { AsGa (BS) } \\
\text { 2.2 Métal-isolant- } \\
\text { métal (MIM) }\end{array}$ & $\begin{array}{c}84 \\
10,6 \\
337 \\
\left\{\begin{array}{c}5\left(^{i}\right) \\
10,6\left(^{i}\right) \\
42 \\
10,6\end{array}\right. \\
\left\{\begin{array}{c}0,5\left(^{i, k}\right) \\
1,52\left({ }^{l}\right)\end{array}\right.\end{array}$ & 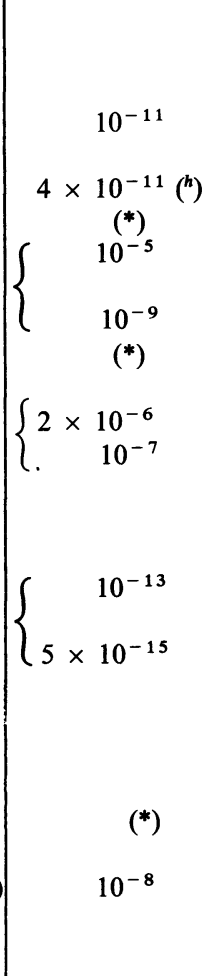 & $\begin{array}{c}28,3 \\
\left(^{*}\right) \\
7,14 \\
\\
2,52 \\
\left(^{*}\right) \\
\\
197 \\
28,3\end{array}$ & $\begin{array}{l}\left\{\begin{array}{c}3,56 \\
(1,58) \\
0,89 \\
0,89\end{array}\right. \\
\left\{\begin{array}{c}4,25 \\
(2,52) \\
28,3\end{array}\right. \\
\left\{\begin{array}{c}197 \\
(88) \\
(10)\end{array}\right.\end{array}$ & $\begin{array}{c}4 \\
23 \\
9 \\
9 \\
1\left(^{j}\right) \\
33 \\
1\left(^{j}\right) \\
1\left(^{(m)}\right. \\
3\left(^{(n)}\right. \\
15\end{array}$ & $\begin{array}{r}1120 \\
1120 \\
235\end{array}$ & $\left\{\begin{array}{c}9 \times 10^{-13} \\
10^{-12} \\
2 \times 10^{-14} \\
5 \times 10^{-17} \\
1 \text { à } 2 \times 10^{-19} \\
10^{-8} \\
10^{-13} \\
10^{-15}-10^{-16}\end{array}\right.$ & $\begin{array}{c}0,89 \\
0,89 \\
0,89 \\
2,52 \\
0,76 \text { à } 0,32 \\
28,3 \\
28,3 \\
0,89\end{array}$ \\
\hline
\end{tabular}

( $\left.{ }^{a}\right)$ NEP : noise equivalent power, représente la mesure de la sensibilité d'un détecteur (non hétérodyne ou incohérent). C'est la puissance de signal pour laquelle le rapport puissance signal/puissance de bruit $=1$, dans une largeur de bande normalisée de $1 \mathrm{~Hz}$.

(b) $M D P$ : minimum detectable power. Ce terme s'applique à un mélangeur ou à un récepteur (en général, c'est le $M D P$ du récepteur que l'on trouve dans le tableau), avec une largeur de bande normalisée de post-détection de $1 \mathrm{~Hz} . M D P=k T B_{\mathrm{F}}, T$ étant la température de bruit du mélangeur (ou du récepteur), $k$ la constante de Boltzmann et $B_{\mathrm{F}}$ la bande de post-détection. La limite quantique est donnée par $h v=k T$.

(c) $v_{1}$ est la fréquence la plus élevée obtenue avec le dispositif.

$\left.{ }^{d}\right)\left(v_{2}\right)$ (il peut y avoir plusieurs valeurs) est la fréquence la plus haute obtenue pour les ordres de multiplication les plus élevés, indiqués en colonne 5 .
$\left({ }^{e}\right) 1 \mathrm{fs}=10^{-15} \mathrm{~s}$
(f) $\mathrm{CP}$ : contact à pointe.
$\left({ }^{g}\right)$ BS : barrière de Schottky.
$\left({ }^{h}\right)$ Valeur estimée.
(i) Redressement supposé d'origine thermique.
(j) Mélange de deux fréquences sans création d'harmonique.
$\left({ }^{k}\right)$ Tension redressée positive.
(l) Tension redressée négative.
$\left({ }^{m}\right)$ Mélange de trois fréquences sans création d'harmoniques.
( $\left.{ }^{n}\right)$ Mélange de trois fréquences avec création d'harmoniques.
$\left({ }^{*}\right)$ Valeur non publiée. 
NEP (1) (estimés) assez faibles (Tableau I). Cette détection a été réalisée à des fréquences bien supérieures à la fréquence de plasma du semiconducteur, qui limite la fréquence d'utilisation de la diode Schottky. Des théories très récentes montrent la possibilité de fonctionnement de ces diodes au-dessus de la fréquence théorique de plasma [43]. Des diodes en tungstène-germanium et en tungstène-arseniure de gallium [44] ont permis des multiplications de fréquence d'ordre 9 à partir de $100 \mathrm{GHz}$, et d'arriver ainsi à $337 \mu \mathrm{m}$ (Tableau I).

Une nouvelle diode à pointes à large bande passante a été décrite dernièrement [45] : la diode à porteurs chauds, constituée par un métal $(\mathrm{AuCu})$ et un semiconducteur (n-InAs). Le rayonnement laser couplé à travers l'antenne chauffe les porteurs du semiconducteur en créant un gradient de température entre les porteurs proches de la pointe et les porteurs " froids » de l'intérieur du semiconducteur. Ceci produit une tension thermoélectrique, fonction du rayonnement reçu. Un mélange entre deux lasers $\mathrm{CO}_{2}$ à $10,6 \mu \mathrm{m}$ a été obtenu avec une fréquence intermédiaire de $54 \mathrm{GHz}$, ce qui montre l'existence d'un phénomène relativement rapide. Pour l'instant, le bruit du récepteur est assez élevé (Tableau I), mais on peut espérer l'améliorer.

Des performances très intéressantes ont été obtenues dans l'infrarouge lointain avec des diodes à barrière Schottky, où la jonction est réalisée par dépôt d'un métal ( $\mathrm{Au}$ ou $\mathrm{Pt}$ ) sur un semiconducteur (n-AsGa). Le diamètre du contact est compris entre $0,25 \mu \mathrm{m}$ et $1,5 \mu \mathrm{m}$. Il est supérieur de plusieurs ordres de grandeur à celui d'un fil épointé en contact avec un métal ou un semiconducteur. Dans ce dispositif, la pointe de l'antenne ne fait pas partie de la jonction, comme c'est le cas dans tous les autres dispositifs à pointes. Le couplage du rayonnement laser à la jonction est assuré par la pointe du whisker, qui appuie sur la partie métallique de la jonction.

Les diodes Schottky sont très largement utilisées comme détecteur entre 42 et $1222 \mu \mathrm{m}$ [46, 47], et comme mélangeurs entre 70 et $1300 \mu \mathrm{m}[34,47,49,50]$ avec des MDP très faibles (Tableau I). Des multiplications de fréquence d'ordre élevé $(\times 33)$ jusqu'à 2,52 $\mathrm{THz}$ ont pu être réalisées [48]. Des mélanges sans création d'harmoniques et à faible fréquence de battement (quelques $\mathrm{MHz}$ ) ont été obtenus à $30 \mathrm{THz}$, en faisant battre deux lasers à $\mathrm{CO}_{2}$ accordés sur la même raie, sur une diode Schottky [51], mais des essais postérieurs [52] n'ont pas confirmé ce résultat. Il semble qu'il s'agisse d'une détection basée sur un effet lent.

La diode Schottky très largement utilisée dans le domaine millimétrique et submillimétrique se caractérise par une bonne fiabilité, une excellente sensibilité et un bruit assez bas avec un MDP situé 100 fois au-dessus de la limite quantique. De plus il peut être

( $\left.{ }^{1}\right)$ Voir définition du $N E P$ et du $M D P$ dans tableau I

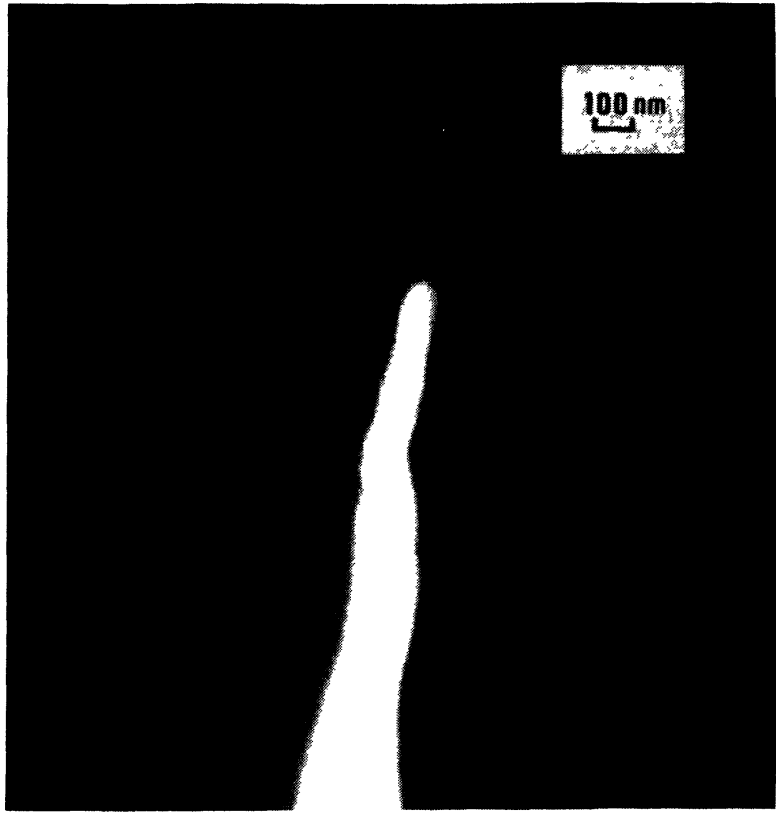

Fig. 8. - Photo d'une pointe en tungstène obtenue au microscope à balayage électronique $(\times 45000)$.

[Electron microscope photograph of the etched tip of a tungsten whisker $(\times 45000)$.

réduit par refroidissement. Elles constituent un élément de choix pour les récepteurs travaillant entre 100 et $1000 \mu \mathrm{m}[48-50,88]$.

4.1.2 La diode métal-isolant-métal (MIM). - Cette diode a été utilisée pour la première fois dans le domaine optique dans le même laboratoire où fut développée la diode MS [53]. En général, une pointe de tungstène de rayon de courbure compris entre 50 et $100 \mathrm{~nm}$ (Fig. 8) appuie sur une base en nickel poli ; entre les deux existe une couche d'oxyde naturel d'épaisseur comprise entre 1 et $2 \mathrm{~nm}$.

Malgré son bruit moyen en détection [57] (Tableau I), sa relative instabilité mécanique et son faible ordre de multiplication, inférieur à 15 , cette diode est la plus largement utilisée à très haute fréquence. Employée comme générateur d'harmoniques, la fréquence la plus élevée mesurée est égale à $88 \mathrm{THz}$ $[23,54,55]$, mais par addition de fréquences, sans génération d'harmoniques, elle a été utilisée pour la mesure du laser à Xe $(2 \mu \mathrm{m})$ à $148 \mathrm{THz}$ [56] et tout dernièrement pour la mesure de la fréquence laser la plus élevée, $197 \mathrm{THz}$ [3], fréquence d'un laser $\mathrm{He}-\mathrm{Ne}$ à $1,52 \mu \mathrm{m}$. Le MDP mesuré pour un récepteur à 10,6 $\mu \mathrm{m}$ est de l'ordre de $10^{-13} \mathrm{~W} \mathrm{~Hz}^{-1}$ [57] et les largeurs de bande des fréquences intermédiaires sont de l'ordre de $50 \mathrm{GHz}$.

Une diode MIM, comme on l'a vu plus haut, est constituée par une pointe métallique très fine, en contact avec un second métal oxydé en surface. Un dispositif mécanique très précis permet de régler la pression sur la pointe et donc l'impédance de la diode. 
Le fonctionnement d'une diode MIM peut être expliqué, au moins en partie, en tenant compte de la conduction des électrons par effet tunnel à travers la très mince barrière (1-2 $\mathrm{nm}$ ) d'oxyde qui constitue l'isolant. L'émission de champ renforcée par effet thermique [58] de la pointe constitue une deuxième possibilité conduisant à la détection de signaux de très haute fréquence. Cette conduction à caractère thermique expliquerait en partie la défaillance actuelle des diodes pour des fréquences proches de $300 \mathrm{THz}$ $(\lambda \simeq 1 \mu \mathrm{m})$.

En effet, pour des fréquences supérieures à environ $100 \mathrm{THz}$, la polarité de la tension redressée (normalement négative avec la base de nickel reliée à la masse dans une combinaison tungstène-nickel $[3,4,59,60])$ tend à s'inverser (c'est-à-dire devient positive) lorsqu'on augmente la fréquence et reste positive jusque dans la zone visible du spectre $(0,5-0,6 \mu \mathrm{m})[59,60]$. Parfois, en augmentant l'impédance en courant conti-
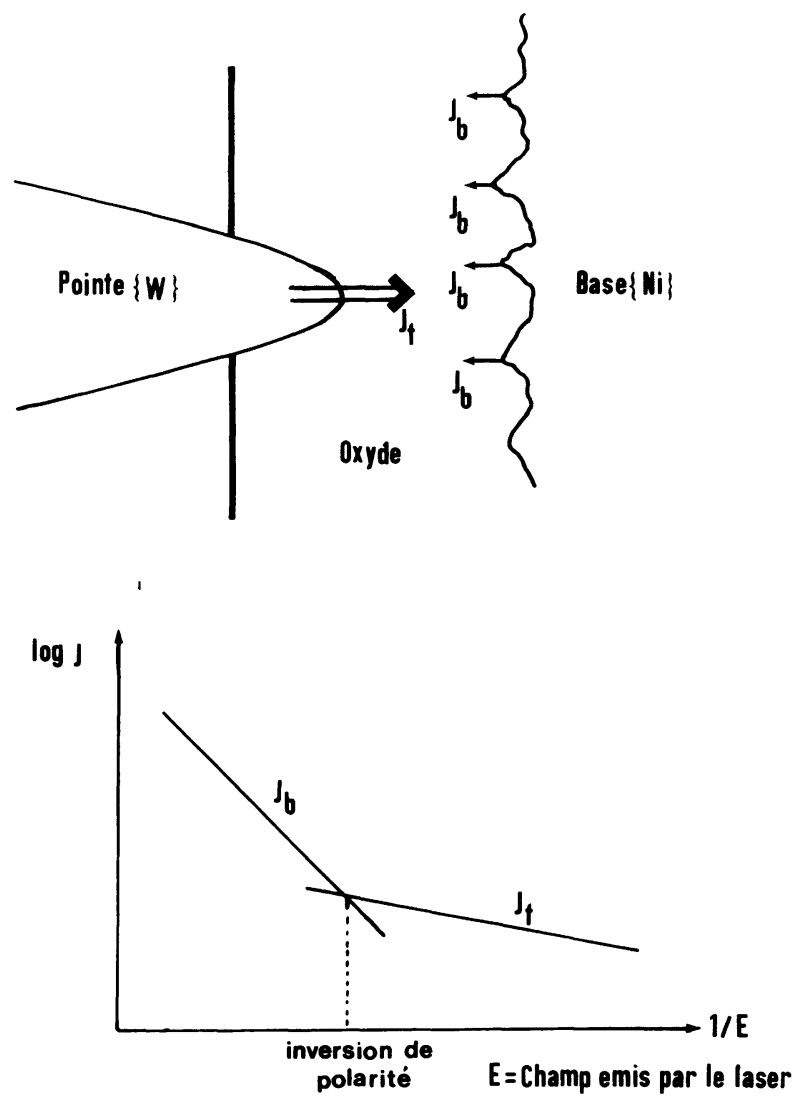

Fig. 9. - Schéma d'une diode MIM dans la zone éclairée par le faisceau laser, avec indication des courants d'émission de la pointe $\left(J_{t}\right)$ et des micro-reliefs de la base $\left(J_{\mathrm{b}}\right)$. Le diagramme inférieur représente l'émission de courant direct et inverse, en tenant compte de la caractéristique d'émission de type Fowler-Nordheim, en fonction du champ émis par le laser E. Une inversion de polarité peut apparaître pour $J_{\mathrm{b}}>J_{\mathrm{t}}$. (D'après réf. [58].)

[Schematic diagram of a MIM diode in the proximity of the laser beam. $J_{\mathrm{t}}$ and $J_{\mathrm{b}}$ are the current densities from the tip and the protusions on the base surfaces respectively. Lower diagram represents the Fowler-Nordheim plots for emission in forward and reverse tip-anode directions respectively, versus the laser emitted field. A polarity reversal may appear for $J_{b}>J_{t}$ (from [58]).] nu de la diode, on peut arriver de nouveau à obtenir un signal redressé négatif $[3,35]\left({ }^{2}\right)$. Ces effets semblent indiquer qu'un nouveau phénomène physique, tel que photoélectrique, thermique ou autre, devient prépondérant et est à l'origine du changement de polarité du signal redressé $\left({ }^{2}\right)$. Expérimentalement, il n'est en général pas possible d'obtenir la génération d'harmoniques à partir d'un signal redressé positif et les signaux de battement les plus grands sont toujours obtenus avec un signal redressé négatif $[3,59]$.

Une explication possible de l'inversion de polarité des diodes MIM a été apportée récemment [58, 62] à partir d'un modèle d'émission de champ renforcé par effet thermique. Cet effet serait dû au réchauffement de la pointe par la puissance du faisceau laser appliqué. Des microreliefs existant sur la surface de nickel, face à la surface active de la pointe en tungstène (Fig. 9), pourraient donner lieu à une émission de courant $J_{\mathrm{b}}$ dans le sens nickel-tungstène plus grande que l'émission de courant normale $J_{t}$ dans le sens tungstène-nickel, en tenant compte des caractéristiques d'émission de type Fowler-Nordheim. $J_{b}$ et $J_{t}$ seraient alors fonction du champ électrique local dû au flux incident provenant du laser et de la température locale des aspérités et de la pointe $\left({ }^{2}\right)$. Ainsi, pour les faibles puissances du laser $J_{\mathrm{b}}$ est inférieur à $J_{\mathfrak{t}}$, tandis que pour les fortes puissances $J_{\mathrm{b}}$ est supérieur à $J_{\mathrm{t}}$, et une inversion de la polarité pourrait apparaître.

Le temps de réponse obtenu expérimentalement, d'une diode MIM, est le plus faible de tous les dispositifs infrarouges; il est de l'ordre de $10^{-14} \mathrm{~s}\left(5 \times 10^{-15} \mathrm{~s}\right.$, si l'on considère comme fréquence limite expérimentale celle de $197 \mathrm{THz}$ mesurée par mélange de fréquences [3]). Théoriquement, la fréquence limite de la diode est fixée par le temps de transit des électrons entre les deux électrodes, mais en fait, la capacité propre de la diode impose une limite beaucoup plus basse $\tau_{R C}, R$ et $C$ étant la résistance et la capacité effectives de la diode [58, 63]. A partir du schéma équivalent de la diode (Fig. 10), le temps de transit fixé par les constantes localisées, est :

$\tau_{R C}=C \frac{r_{\mathrm{a}} R}{r_{\mathrm{a}}+R}$

où $r_{\mathrm{a}}$ est la résistance équivalente de l'antenne. Ceci conduit à des valeurs typiques

$\tau_{R C} \simeq 10^{-14} \mathrm{~s} \quad\left(f_{\mathrm{c}} \simeq 100 \mathrm{THz}\right)$

pour des rayons de courbure de la pointe de quelques dizaines de $\mathrm{nm}$. Pour un modèle de conduction par effet tunnel, le temps de passage à travers une barrière

( ${ }^{2}$ ) L'effet d'inversion de polarité a été étudié théoriquement et expérimentalement dans une jonction $\mathrm{Al}-\mathrm{Al}_{2} \mathrm{O}_{3}-\mathrm{W}$ soumise à un signal micro-ondes [61]. L'inversion de polarité est obtenue pour des signaux à fort niveau et elle dépend de la résistance de la jonction et de la fonction travail des métaux utilisés. 


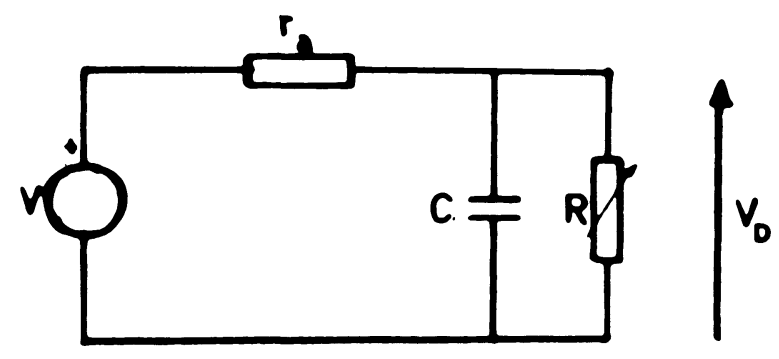

Fig. 10. - Schéma équivalent de la diode MIM à constantes localisées.

[Lumped circuit of a MIM diode.]

dont l'épaisseur est de l'ordre de 1-2 nm, typique pour une diode MIM à oxyde, serait donné par $[58,60,64]$

$t \simeq \frac{h}{\left(E_{\mathrm{f}} \varphi\right)^{1 / 2}}$

où $h$ est la constante de Planck, $\varphi$ la hauteur de la barrière entre métal et isolant, et $E_{\mathrm{r}}$ l'énergie des électrons au niveau de Fermi. Pour une jonction tungstène-nickel, ce temps de passage serait $t \simeq 10^{-16} \mathrm{~s}$, ce qui correspond à une fréquence de $10000 \mathrm{THz}$ (30 nm). Ainsi, ce modèle donne des valeurs du temps de transit de deux ordres de grandeur supérieurs à celui du modèle $\mathrm{RC}$.

La capacité de la jonction limitant la fréquence de coupure de la diode, il semble intéressant, pour monter en fréquence, de développer une diode MIM où le diélectrique serait le vide $\left(\varepsilon_{\mathrm{r}}=1\right.$ au lieu de $\varepsilon_{\mathrm{r}}=4$ à 8 dans l'isolant) et dont le fonctionnement serait fondamentalement dû à l'émission de champ. Ceci pourrait permettre d'augmenter la fréquence de coupure de la diode jusqu'au domaine du visible.

4.1.3 La diode supraconducteur-isolant-supraconducteur (jonction Josephson). - Les faibles températures de fonctionnement des jonctions Josephson font que ce dispositif est de très loin le meilleur en ce qui concerne le bruit $\left(N E P \simeq 10^{-13} \mathrm{~W} \mathrm{~Hz}^{-1 / 2}\right.$ à $78 \mu \mathrm{m}$ [65] et $M D P=6 \times 10^{-20} \mathrm{~W} \mathrm{~Hz}^{-1}$ pour un récepteur à $0,66 \mu \mathrm{m}$ [66]). Ces valeurs sont supérieures d'un facteur 10 à 100 à la limite quantique et semblent être limitées par le bruit de l'amplificateur utilisé en fréquence intermédiaire.

Les jonctions utilisées dans les domaines millimétriques et submillimétriques sont réalisées en niobium-oxyde de niobium-niobium, et les pointes ont un diamètre de quelques micromètres, qui est ainsi supérieur de un à deux ordres de grandeur à ceux des contacts utilisés pour les diodes à barrière Schottky ou pour les autres diodes à pointes.

La jonction Josephson a permis d'obtenir les ordres de multiplication de fréquence les plus élevés : 401 à partir de $9,5 \mathrm{GHz}$ pour aboutir à $3,8 \mathrm{THz}[65]$ et 825 à partir de $1 \mathrm{GHz}$ pour atteindre $0,89 \mathrm{THz}$ [67], mais son utilisation reste limitée dans le domaine de l'infrarouge lointain.
La fréquence limite d'emploi d'un supraconducteur est une fonction croissante de la largeur de la bande interdite du supraconducteur, et donc de sa température critique [15]. Pour le niobium $\left(T_{\mathrm{c}}=9,2 \mathrm{~K}\right)$ la fréquence limite est ainsi de $720 \mathrm{GHz}$. Pour des supraconducteurs à haute température critique, tels que le $\mathrm{Nb}_{3} \mathrm{Sn}\left(T_{\mathrm{c}}=18,2 \mathrm{~K}\right)$ et le $\mathrm{Nb}_{3} \mathrm{Ge}\left(T_{\mathrm{c}} \simeq 21 \mathrm{~K}\right)$, la fréquence associée à la largeur de la bande interdite serait de l'ordre de 1,6 THz. D'autres théories (pic de Riedel $[68,69]$ ou phénomènes de relaxation dans le supraconducteur [70]) donnent comme fréquences limites, pour une jonction Josephson en niobium, $1,4 \mathrm{THz}$ et 7,5 $\mathrm{THz}$, respectivement.

Les jonctions à pointes $\mathrm{Nb}-\mathrm{Nb}$ ont permis à ce jour des mélanges à des fréquences supérieures aux fréquences limites obtenues à partir de la largeur de la bande interdite ou du pic de Riedel, mais inférieures à celles prévues à partir des phénomènes de relaxation : $2,53 \mathrm{THz}(118 \mu \mathrm{m})[71] ; 3,8 \mathrm{THz}(78 \mu \mathrm{m})$ [65] et $4,25 \mathrm{THz}(70 \mu \mathrm{m})$ [27], fréquences obtenues par multiplication à partir de générateurs micro-ondes. Des mélanges ont été obtenus à $31,5 \mathrm{THz}(9,5 \mu \mathrm{m})$ [72] en faisant battre dans une jonction Josephson deux lasers à $\mathrm{CO}_{2}$. Ce mélange, obtenu à une fréquence 45 fois supérieure à la fréquence limite due à la largeur de la bande interdite du supraconducteur, pourrait être d'origine thermique, donc à bande passante étroite, et non utilisable pour une synthèse de fréquence à large bande passante. Pour cette raison, nous n'envisageons plus l'utilisation d'une jonction Josephson au niveau du mélange $\mathrm{CO}_{2}-\mathrm{CO}_{2}(\S 5$, Fig. 12) comme il était prévu antérieurement [2, 23, 73]. Malgré cela, les jonctions Josephson sont des dispositifs de choix pour les récepteurs millimétriques et submillimétriques à très faible bruit $[32,34,65,71,74,75,88]$, malgré la servitude imposée par l'emploi de l'hélium liquide. De ce fait, le LPTF $[2,23,73,76]$ et le $N B S$ $[77,78]$ envisagent leur utilisation dans la chaîne de fréquences laser ( $\S 5$, Fig. 12) pour synthétiser directement les 4,25 $\mathrm{THz}(70,5 \mu \mathrm{m})$ d'un laser à alcool méthylique à partir d'un oscillateur à cavité supraconductrice, avec un ordre de multiplication de 425 . Le $N P L$ [27] envisage une synthèse similaire, mais avec un facteur de multiplication dix fois inférieur, à partir d'un klystron à $100 \mathrm{GHz}$.

Des études sont en cours [27, 70,77] pour déterminer la fréquence ultime d'utilisation d'une jonction Josephson, qui peut être limitée par la capacité de la jonction, de l'ordre de $10^{-13} \mathrm{~F}$, et par des effets thermiques dus aux niveaux de puissance injectée. D'autres études portent sur le niveau de puissance obtenu en fréquence intermédiaire, qui varie en $N^{-2}, N$ étant le facteur de multiplication utilisé.

4. 2 Les diodes A STRUCTURe Planar. - Deux types de diodes à structure planar ont été réalisés à ce jour : des diodes Schottky et des diodes MIM.

Bien que les fréquences de fonctionnement comme mélangeur des deux dispositifs soient actuellement 
relativement basses, à cause de la forte capacité due à la jonction planar, elles peuvent seules permettre dans le futur (après amélioration des techniques de miniaturisation) l'intégration de plusieurs centaines de dispositifs sur la même pastille, pour obtenir des niveaux de puissance plus élevés, et un meilleur couplage de la diode au rayonnement incident [79]. D'autre part, les avantages provenant d'une meilleure stabilité mécanique et d'une reproductibilité améliorée, sont également importants.

4.2.1 La diode à barrière Schottky. - Des diodes avec des diamètres de contact de l'ordre de $2 \mu \mathrm{m}$ ont fonctionné dans le domaine des longueurs d'onde submillimétriques (LIR) [80, 81].

Des détections jusqu'à $337 \mu \mathrm{m}$ ont été obtenues [81], ainsi que des mélanges avec des ordres de multiplication très élevés : 82 à partir de $9,3 \mathrm{GHz}$ (pour arriver à $762 \mathrm{GHz}$ [80] et 9 à partir de $74,2 \mathrm{GHz}$ (pour arriver à $667 \mathrm{GHz}$ ) [81].

4.2.2 La diode MIM plane. - Des contacts MIM planar évaporés $\left(\mathrm{Al}-\mathrm{Al}_{2} \mathrm{O}_{3}-\mathrm{Al}\right.$; Ni-NiO-Ni : $\mathrm{Cr}-\mathrm{NiO}-$ Ni) [82-85] avec des surfaces de $10^{-6}$ à $10^{-10} \mathrm{~cm}^{2}$ (10 à 10000 fois plus grandes que dans le cas d'une diode à pointe) ont permis des mélanges à $337 \mu \mathrm{m}$, avec des ordres de multiplication assez élevés $(70 \mathrm{GHz} \times 13)$ [82] (Tableau I).

Des détections entre 0,45 et $0,6 \mu \mathrm{m}$ [83-85] sont attribuées à des électrons photo-induits à travers la barrière [83] ou à des effets thermiques [84, 85]. Des détections à $10,6 \mu \mathrm{m}[82,85]$ et $3,39 \mu \mathrm{m}$ [85] ont également été obtenues.

Les jonctions à faible surface $\left(10^{-10} \mathrm{~cm}^{2}\right)$ [85] pourront peut-être permettre le mélange des fréquences infrarouges dans un proche avenir.

5. Chaînes de synthèse des fréquences optiques. Les dispositifs précédemment décrits (lasers et diodes) fonctionnant entre les micro-ondes et l'infrarouge proche, ont permis la comparaison successive des fréquences d'une chaîne d'oscillateurs jusqu'à $1,52 \mu \mathrm{m}$ [3].

Pour pouvoir mesurer une fréquence laser très élevée, celle par exemple à $88 \mathrm{THz}$ du laser $\mathrm{He}-\mathrm{Ne}$ $\left(\mathrm{CH}_{4}\right)$, il est nécessaire de mettre en place une véritable chaîne d'oscillateurs ayant des fréquences intermédiaires entre celle de l'étalon primaire, l'horloge à césium à laquelle il faut relier la mesure, et celle à mesurer. On effectue alors la mesure des différentes fréquences intermédiaires en allant des plus basses vers les plus élevées, chaque mesure s'appuyant sur la mesure précédente. Il est évident que la précision atteinte se dégrade lorsqu'on se rapproche du visible, les erreurs dues aux instabilités non corrélées des sources s'additionnant.

La figure 11 montre le principe de la chaîne de synthèse des fréquences lasers utilisée au NBS (USA) et au NPL (GB) et actuellement en cours de réalisation au LPTF avec la collaboration des Universités de

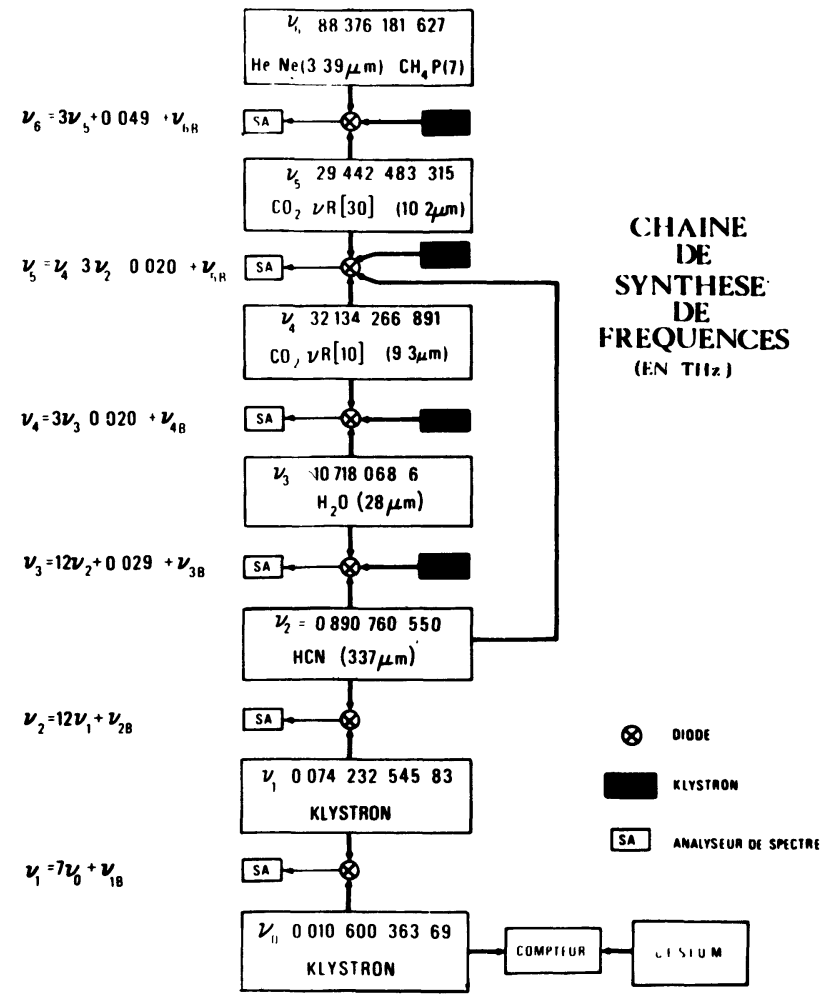

Fig. 11. - Principe de la chaîne de raccordement des fréquences optiques $(88 \mathrm{THz})$ aux fréquences radioélectriques (césium).

[Classical laser frequency synthesis chain from the cesium frequency standard to the $\mathrm{He}-\mathrm{Ne}(88 \mathrm{THz})$ laser.]

Paris-VI et Paris-XI [2, 4, 23, 73]. Les lasers $\mathrm{He}-\mathrm{Ne}$ $\left(\mathrm{CH}_{4}\right)$ et $\mathrm{CO}_{2}\left(\mathrm{CO}_{2}\right)$ sont d'excellents étalons secondaires de fréquence $(\S 2)$ stables et reproductibles. Ceci n'est pas le cas pour les lasers de transfert à $\mathrm{HCN}$ et $\mathrm{H}_{2} \mathrm{O}$, dont la largeur de raie est de l'ordre de quelques $\mathrm{kHz}$ ou dizaines de $\mathrm{kHz}$.

Malgré ceci, des mesures de la fréquence du laser $\mathrm{He}-\mathrm{Ne}\left(\mathrm{CH}_{4}\right)$ ont été réalisées en utilisant cette méthode de synthèse par le $N B S$ et le $N P L$. Les valeurs obtenues sont respectivement :

$$
\begin{aligned}
& v_{\mathrm{CH}_{4}}=88376181,627 \pm 0,050 \mathrm{MHz}[54] \\
& v_{\mathrm{CH}_{4}}=88376181,608 \pm 0,043 \mathrm{MHz}[55]
\end{aligned}
$$

ce qui correspond à des précisions de $6 \times 10^{-10}$ et $5 \times 10^{-10}$ respectivement, sur la valeur de la fréquence.

Afin de réduire le nombre de lasers utilisés actuellement et d'obtenir une meilleure précision sur la mesure, de l'ordre de $10^{-12}$, une nouvelle chaîne de synthèse de fréquence est actuellement en cours de réalisation au $N B S[77,78]$ et au LPTF $[2,4,23,73,90]$ (Fig. 12). Dans cette chaîne, un oscillateur à cavité supraconductrice sera utilisé à la place du klystron, à une fréquence voisine de $10 \mathrm{GHz}$, pour profiter de la grande pureté spectrale de cet oscillateur ( $(2)$.

Etant donné l'ordre de multiplication élevé entre le SCSO et le premier laser (alcool méthylique, 


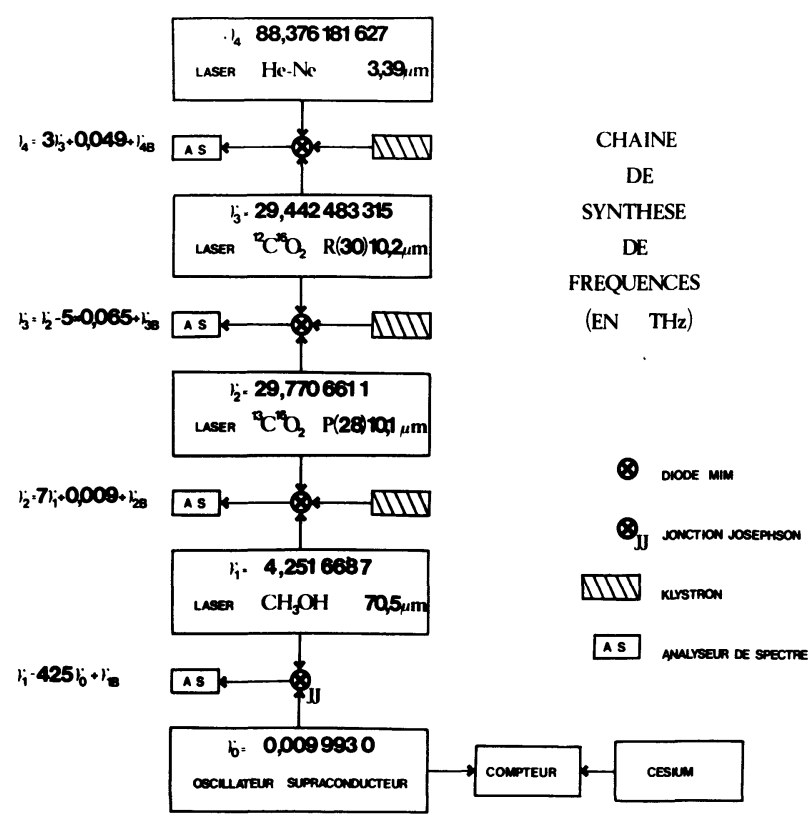

Fig. 12. - Principe de la nouvelle chaîne de raccordement des fréquences optiques $(88 \mathrm{THz})$ aux fréquences radioélectriques (césium).

[New laser frequency synthesis chain from the cesium frequency standard to the $\mathrm{He}-\mathrm{Ne}(88 \mathrm{THz})$ laser.]

$\mathrm{CH}_{3} \mathrm{OH}$ ), seule une jonction Josephson peut être utilisée (§ 4.1.3).

Le premier laser de la chaîne est un laser à $\mathrm{CH}_{3} \mathrm{OH}$, pompé optiquement par un laser à $\mathrm{CO}_{2}$, et travaillant à $70,5 \mu \mathrm{m}(4,25 \mathrm{THz})$. Ce laser, à guide d'onde, est particulièrement intéressant, car il n'utilise pas un plasma comme c'est le cas des lasers à décharge $\left(\mathrm{He}-\mathrm{Ne}, \mathrm{CO}_{2}, \mathrm{H}_{2} \mathrm{O}, \mathrm{HCN}\right)$. Il est donc intrinsèquement moins bruyant que les lasers à gaz classiques.

La figure 13 montre une comparaison préliminaire $[4,28]$ entre la stabilité de fréquence, caractérisée par la racine carrée de la variance d'Allan, $\sigma_{y}(\tau)$, des différents lasers IR et LIR non asservis : deux lasers à décharge, $\mathrm{HCN}$ [86] et $\mathrm{CO}_{2}$, et celle du laser $\mathrm{CH}_{3} \mathrm{OH}$. Pour $\tau \leqslant 0,1$ les lasers $\mathrm{CO}_{2}$ et $\mathrm{HCN}$ présentent un bruit flicker de fréquence (variation de l'écart-type des fluctuations de fréquence en $\tau^{0}$ ) et pour $\tau>0,1 \mathrm{~s}$ (laser $\mathrm{CO}_{2}$ ) et $\tau>1 \mathrm{~s}$ (laser $\mathrm{HCN}$ ), une dérive de fréquence due essentiellement à des effets thermiques et aux variations de courant de la décharge. Le laser $\mathrm{CH}_{3} \mathrm{OH}(70,5 \mu \mathrm{m})$ a un bruit blanc de fréquence, dont l'écart-type des fluctuations de fréquence varie comme $\tau^{-1 / 2}$ pour $\tau \leqslant 0,1 \mathrm{~s}$. Pour $0,1 \mathrm{~s}<\tau \leqslant 1 \mathrm{~s}$, le laser $\mathrm{CH}_{3} \mathrm{OH}$ présente une dérive de fréquence.

Des stabilités similaires ont été obtenues dernièrement au $P T B$ (RFA) pour un laser $\mathrm{HCOOH}$ à $394 \mu \mathrm{m}$ (761 GHz) [87].

Nous avons étudié dernièrement la stabilité de fréquence du laser $\mathrm{CH}_{3} \mathrm{OH}$ à $70,5 \mu \mathrm{m}$ en faisant battre dans une diode MIM deux lasers libres pompés par le même laser $\mathrm{CO}_{2}$. Les résultats obtenus en utilisant un amplificateur de post-détection à large bande

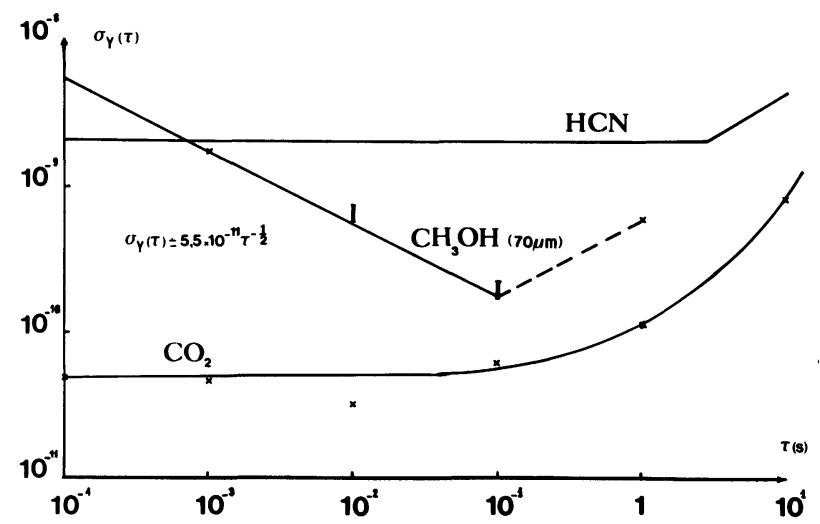

Fig. 13. - Comparaison de la stabilité de fréquence des lasers libres à décharge, $\mathrm{HCN}$ [86] et $\mathrm{CO}_{2}$, avec un laser sans décharge $\mathrm{CH}_{3} \mathrm{OH}(70,5 \mu \mathrm{m})[4,28]$. La courbe de stabilité du laser $\mathrm{CH}_{3} \mathrm{OH}$ a été obtenue par battement de deux lasers $(2 \mathrm{~m}$ et $4 \mathrm{~m}$ de long) pompés par le même laser $\mathrm{CO}_{2}$ libre. Signal de battement obtenu : $2 \mathrm{MHz} ; S / B=60 \mathrm{~dB}$.

[Comparison of the free-running frequency stability of two gas discharge lasers, $\mathrm{HCN}\left[86\right.$ and $\mathrm{CO}_{2}$ pump laser, with an opticallypumped $\mathrm{CH}_{3} \mathrm{OH}(70.5 \mu \mathrm{m})$ laser $[4,28]$. The $\mathrm{CH}_{3} \mathrm{OH}$ stability curve has been obtained from two ( 2 and $4 \mathrm{~m}$ long) lasers pumped by the same free runing $\mathrm{CO}_{2}$ laser. Characteristics of the $\mathrm{CH}_{3} \mathrm{OH}$ beat note are $: v_{\mathrm{B}}=2 \mathrm{MHz}, S / N=60 \mathrm{~dB}$.]

$(400 \mathrm{MHz})$ sont similaires à ceux de la figure $13[88,89]$. L'utilisation d'un amplificateur de post-détection à bande étroite $(5 \mathrm{MHz})$ a permis d'améliorer de deux ordres de grandeur la mesure de la stabilité de fréquence du laser pompé [88-90]: $\sigma_{y}(\tau)=2 \times 10^{-13} \tau^{-1 / 2}$ (bruit blanc de fréquence) pour $\tau \leqslant 20 \mathrm{~ms}$, avec une valeur minimale $\sigma_{y}(50 \mathrm{~ms})=2 \times 10^{-12}$. Pour des temps de mesure supérieurs, une dérive de fréquence apparaît $\sigma_{y}(\tau) \simeq 1,5 \times 10^{-11} \tau$, due probablement à la dérive de la cavité LIR et à celle du laser $\mathrm{CO}_{2}$ de pompe.

La figure 14 montre une comparaison de la stabilité de fréquence de deux lasers LIR : le meilleur laser HCN asservi en phase [86] et le laser $\mathrm{CH}_{3} \mathrm{OH}(70,5 \mu \mathrm{m})$ non asservi, dont l'écart-type de fluctuations de fréquence est mesuré à travers un amplificateur à bande étroite [88-90].

Des mesures sont en cours pour déterminer l'influence de la stabilité du laser de pompe sur celle du laser LIR [89].

La grande stabilité de fréquence du laser $\mathrm{CH}_{3} \mathrm{OH}$ $(70,5 \mu \mathrm{m})$ non asservi et sa faible largeur de raie (des valeurs préliminaires de l'ordre de $400 \mathrm{~Hz}$ ont été obtenues), permettent d'envisager son utilisation dans la chaîne de synthèse de fréquence (Fig. 12) sans détériorer la pureté spectrale de l'oscillateur supraconducteur, après multiplication [90].

Pour réduire les erreurs dues aux instabilités des sources, nous essayons d'asservir en phase la chaîne des lasers. Ainsi, en asservissant sur l'étalon de fréquence tous les oscillateurs de la chaîne jusqu'au visible, la fréquence de la dernière source aura à long terme la même stabilité que l'étalon. 


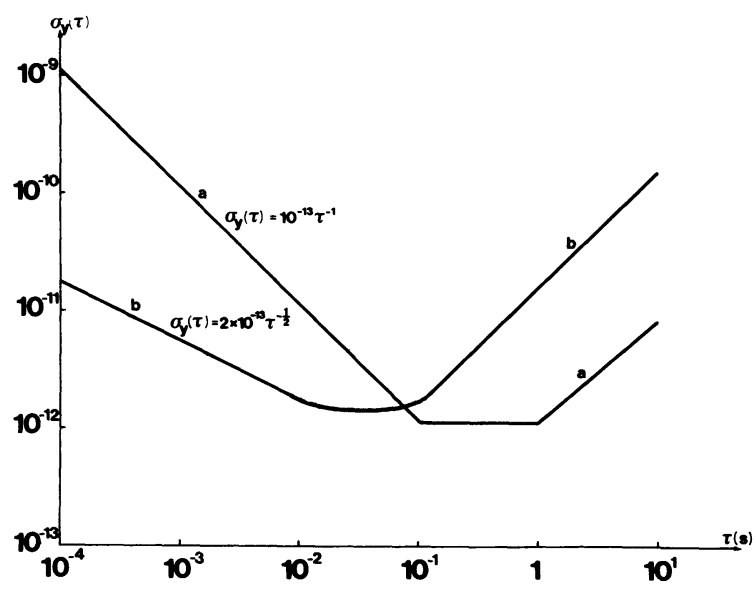

Fig. 14. - Comparaison de la stabilité de fréquence, $\sigma_{y}(\tau)$, des lasers LIR les plus stables : $a$ ) le laser $\mathrm{HCN}(337 \mu \mathrm{m})$ asservi en phase [86] et $b$ ) le laser $\mathrm{CH}_{3} \mathrm{OH}(70,5 \mu \mathrm{m})$ non asservi [88-90]. La courbe de stabilité du laser $\mathrm{CH}_{3} \mathrm{OH}$ a été obtenue par battement de deux lasers similaires ( $2 \mathrm{~m}$ de long), pompés par le même laser $\mathrm{CO}_{2}$ libre. Signal de battement obtenu : $180 \mathrm{kHz}, S / B=50 \mathrm{~dB}$

[Compared fractional frequency stability, $\sigma_{y}(\tau)$, of the two more stable FIR lasers : $a$ ) the phase-locked HCN laser $(337 \mu \mathrm{m})[86$ and $b$ ) the free-running $\mathrm{CH}_{3} \mathrm{OH}(70,5 \mu \mathrm{m})$ pumped laser [88-90]. The $\mathrm{CH}_{3} \mathrm{OH}$ stability curve has been obtained from two similar $\left(2 \mathrm{~m}\right.$ long) lasers pumped by the same free-running $\mathrm{CO}_{2}$ laser. Characteristics of the $\mathrm{CH}_{3} \mathrm{OH}$ beat note are : $v_{\mathrm{B}}=180 \mathrm{kHz}$; $S / N=50 \mathrm{~dB}$.]

Pour y parvenir, le laser $\mathrm{CH}_{3} \mathrm{OH}$ sera un élément de choix, bien meilleur que le laser $\mathrm{HCN}$, et supérieur au laser $\mathrm{H}_{2} \mathrm{O}$, difficile à asservir en phase.

L'effet Stark permet de moduler très rapidement un laser LIR, en appliquant un champ électrique transversal sur le milieu laser $[19,20,78]$. Cette méthode a l'avantage de ne pas introduire de pertes dans la cavité laser, et la vitesse de modulation est seulement limitée par le temps de vie des photons à l'intérieur de la cavité, de l'ordre de $10 \mu \mathrm{s}$. L'application de ce phénomène au laser $\mathrm{CH}_{3} \mathrm{OH}$ doit permettre d'asservir en phase ce laser sur l'oscillateur supraconducteur, et d'obtenir ainsi, pour la première fois, un étalon de fréquence secondaire dans le LIR, nécessaire à la mesure de la fréquence du laser $\mathrm{He}-\mathrm{Ne}\left(\mathrm{CH}_{4}\right)$ avec une précision de l'ordre de $10^{-12}$.

La fréquence de résonance de l'oscillateur supraconducteur (Fig. 12) a été choisie pour obtenir une fréquence intermédiaire à la sortie de la jonction Josephson capable d'être amplifiée convenablement dans un amplificateur MESFET refroidi à faible bruit (bande de 4,5 à $5 \mathrm{GHz}$ ).

La partie supérieure de la chaîne de synthèse des fréquences laser (Fig. 12) reste inchangée par rapport à celle de la figure 11 , à l'exception du premier laser à $\mathrm{CO}_{2}$ qui émettra la raie $\mathrm{P}_{\mathrm{II}}(28)$ à $10,1 \mu \mathrm{m} \mathrm{du}{ }^{13} \mathrm{C}^{16} \mathrm{O}_{2}$. D'autres raies, telle que $\mathrm{R}_{\mathrm{I}}(50)$ du ${ }^{12} \mathrm{C}^{16} \mathrm{O}_{2}$ [91] auraient donné une fréquence de battement proche de celle qu'on doit obtenir avec la combinaison proposée, mais la première se trouvant au centre du spectre d'émission $d{ }^{13} \mathrm{C}^{16} \mathrm{O}_{2}$ doit permettre d'obtenir des puissances plus élevées. Des expériences pour obtenir la synthèse $\mathrm{CH}_{3} \mathrm{OH}-\mathrm{CO}_{2}$ avec un facteur de multiplication égal à 7 ont été réalisées avec succès au NBS [92] et au PTB [91].

Pour éviter l'utilisation d'une jonction Josephson au niveau du mélange $\mathrm{CO}_{2}-\mathrm{CO}_{2}$, comme il a été proposé antérieurement $[23,73]$, une source micro-ondes de fréquence élevée $(65 \mathrm{GHz})$ et une diode MIM seront utilisées. Ceci doit permettre un meilleur temps de réponse du mélangeur, puisque la jonction Josephson semble limitée à cause des effets thermiques à $10 \mu \mathrm{m}(\S 4.1 .3)$. Un asservissement de phase est prévu sur le $\mathrm{CO}_{2}$ à partir du laser $\mathrm{CH}_{3} \mathrm{OH}$, lui-même asservi sur le SCSO. Ensuite, le laser $\mathrm{He}-\mathrm{Ne}\left(\mathrm{CH}_{4}\right)$ devra être asservi sur le $\mathrm{CO}_{2}$.

Pour améliorer la stabilité du laser à $\mathrm{CO}_{2}$, de nouveaux lasers scellés et sans fenêtres sont à l'étude. Ceci doit permettre une meilleure stabilisation à long terme, seulement limitée par les variations de dimensions de la structure mécanique et par celles du courant de la décharge. Une stabilisation améliorée par fluorescence saturée devra être employée, en utilisant des détecteurs à grande surface et à détectivité élevée, associés à un réflecteur de rayonnement porté à la température de l'azote liquide [7].

Nous espérons ainsi améliorer la précision de la mesure du laser $\mathrm{He}-\mathrm{Ne}\left(\mathrm{CH}_{4}\right)$ d'un facteur 100 environ. Ainsi la voie sera ouverte pour des mesures dans le visible, mais ces mesures ne seront possibles que si des progrès considérables sont réalisés sur les dispositifs non linéaires, cristaux non linéaires [26] ou diodes MIM à émission de champ (§ 4.1.2) pouvant être utilisés dans cette gamme de fréquence.

\section{Bibliographie}

[1] Audoin, C., Vanier, J., J. Phys. E 9 (1976) 697-720.

[2] Jimenez, J. J., "Les étalons de fréquence laser ». Presidencia del Gobierno. Comision Nacional de Metrologia y Microtecnia. Madrid (1977).

[3] Evenson, K. M., Jennings, D. A., Petersen, F. R., Wells, J. S., Laser Spectroscopy III, ed. J. L. Hall et J. L. Carlsten (Springer-Verlag) 1977, 56-68.

[4] Jimenez, J. J., Petersen, F. R., Infrared Phys. 17 (1977) 541-546.
[5] Evenson, K. M., Petersen, F. R., Laser spectroscopy of atoms and molecules, ed. H. Walther (Springer-Verlag) 1976, 349-368.

[6] Cerez, P., Brillet, A., Man, C., Revue Phys. Appl. (Ce même numéro.)

[7] Freed, C., O'Donnell, R. G., Proc. 2nd Frequency standards and metrology symposium, Copper Mountain, Colorado, USA, 5-7 juil. 1976, 279-300. 
[8] Clatron, A., Thèse $3^{e}$ cycle, Paris, juin 1974.

[9] Ouhayoun, M., Borde, C. J., Proc. 2nd Frequency standards and metrology symposium, Copper Mountain, Colorado, USA, 5-7 juil. 1976, 145-151.

[10] van Lerberghe, A., Avrillier, S., Borde, C. J., « High stability $\mathrm{CW}$ waveguide $\mathrm{CO}_{2}$ laser for high resolution saturation spectroscopy ", à paraître IEEE J. Quant. Electr., juillet (1978).

[11] Chebotayev, V. P., Proc. 2nd Frequency standards and metrology symposium, Copper Mountain, Colorado, USA, 5-7 juil. 1976, 385-423.

[12] Cerez, P., Bennet, S. J., « New developments in iodine stabilized He-Ne lasers ", CPEM'78 Conf., Ottawa, Canada, 26-29 juin 1978.

[13] Camy, G., Thèse, à paraître, 1978. Voir également article Métrologie, à paraître 1978 .

[14] Stein, S. R., Turneaure, J. P., Proc. IEEE, 63 (1975) 1249 1250.

[15] Septier, A., Viet, N. T., J. Phys. E (GB), 10 (1977) 1193-1207.

[16] JimeneZ, J. J., Thèse d'Etat, Orsay, juin 1974.

[17] Stein, S., Thèse Ph. D, Stanford, septembre 1974.

[18] Benard, J., Communication privée, 1978.

[19] Stein, S. R., van de Stadt, H., Proc. 31st An. Symp. Frequency Control, ed. Electronic Industries Assoc., Washington, DC, 1977, 601-604.

[20] Inguscio, M., Minguzzi, P., Strumia, F., Tonelli, M., 3rd Int. Conf. on Submillimetric waves and their applications, University of Surrey (GB), 29.3-1.4.1978, papier TB2.7.

[21] Petersen, F. R., Evenson, K. M., Jennings, D. A., Wells, J. S., Goto, K., JimeneZ, J. J., IEEE J. Quant. Electr. QE-11 (1975) 838-843.

[22] Jimenez, J. J., Petersen, F. R., « Frequency and linewidth measurement of the CW He-Ne laser at $88 \mathrm{THz}(3.39 \mu \mathrm{m})$ )". A paraître.

[23] Jimenez, J. J., Petersen, F. R., Plainchamp, P., Sallot, C., DraGo, X., Bull. d'Information du BNM, 9 (1978) 19-27.

[24] Petersen, F. R., McDonall, D. G., CupP, J. D., Danielson, B. L., Laser spectroscopy, ed. R. G. Brewer et A. Mooradian (Plenum Press) 1975, 555-569.

[25] Matarrese, L. M., Evenson, K. M., Appl. Phys. Lett. 17 (1970) 8-10.

[26] Petersen, F. R., Communication privée, 1977, 1978.

[27] Knigth, D. J. E., Communication privée, 1978.

[28] Evenson, K. M., Day, G. W., Wells, J. S., Mullen, L. O., Appl. Phys. Lett. 20 (1972) 133-134.

[29] Freed, C., Ross, A. H. M., O'Donnell, R. G., J. Mol. Spectrosc. 49 (1974) 439-453.

[30] Freed, C., O'Donnell, R. G., Ross, A. H. M., IEEE Trans. Instr. Measur. IM-25 (1976) 431-437.

[31] Freed, C., Proc. 31st An. Symp. Frequency Control, ed. Electronic Industries Assoc., Washington, DC, 1977, 592-600.

[32] Knight, D. J. E., Woods, P. T., J. Phys. E 9 (1976) 898-916.

[33] Pyee, M., Auvray, J., Bull. Information BNM 6 (1975) 11-22.

[34] McColl, M., Proc. Soc. Photo-Opt. Instrum. Engrs. (SPIE) 105, Far Infrared Submillimeter Wave, Technology and Applications, 24-34.

[35] Evenson, K. M., Jennings, D. A., Petersen, F. R., Travail non publié.

[36] Mizuno, K., Kuwahara, R., Ono, S., Appl. Phys. Lett. 26 (1975) 605-607.

[37] Hwang, T. L., Schwarz, S. E., Appl. Phys. Lett. 31 (1977) 101-104.

[38] Bolomey, J. C., Rap. LSE no RC 77-8 CNRS, E.S.E., 1977, et publication à paraître

[39] Rutledge, D. B., Schwarz, S. E., 3rd Int. Conf. Submillimetric Waves and their Applications, Université de Surrey (GB), 29.3-1.4.1978, papier FC 1.6.

[40] Krautle, H., Sauter, E., Schultz, G. V., Ibid., papier FC 1.7. Voir aussi Infrared Phys., 17 (1977) 477-483.
[41] Hocker, L. O., Javan, A., RaO, D. R., Frenkel, L., Sullivan, T., Appl. Phys. Lett. 10 (1967) 147-149.

[42] Tsang, D., Schwarz, S. E., Appl. Phys. Lett. 30 (1977) 263265.

[43] Champlin, K. S., Eisenstein, G., Appl. Phys. Lett. 31 (1977) 221-223.

[44] Zuidberg, B. F., Dymanus, A., Appl. Phys. Lett. 29 (1976) 643-645.

[45] Aukerman, L. W., Erler, J. W., Opt. Lett. 1 (1977) 178-180.

[46] McColl, M., Hodges, D. T., GARber, W. A., IEEE Trans. Microw. Theory Techn. MTT-25 (1977) 463-467.

[47] Hodges, D. T., McColl, M., Appl. Phys. Lett. 30 (1977) 5-7.

[48] Fetterman, H. R., Clifton, B. J., Tannenwald, P. E., Parker, C. D., Appl. Phys. Lett. 24 (1974) 70-72.

[49] Fetterman, H. R., Tannenwald, P. E., Clifton, B. J., Parker, C. D., Fitzgerald, W. D., Erickson, N. R., " High sensitivity submillimetric heterodyne receiver". A paraître IEEE Trans. Microw. Theory Techn., 1978.

[50] McColl, M., Hodges, D. T., Chase, A. B., Garber, W. A., Proc. 31st An. Symp. Frequency Control, ed. Electronic Industries Assoc., Washington, DC (1977) 574-577.

[51] Pyee, M., Clairon, A., Auvray, J., Electr. Lett. 10 (1974) 500-501.

[52] Fetterman, H. R., Communication privée, 1976.

[53] Hocker, L. O., Javan, A., Phys. Lett. 26A (1968), 255-256.

[54] Evenson, K. M., Wells, J. S., Petersen, F. R., Danielson, B. L., DAY, G. W., Appl. Phys. Lett. 22 (1973) 192-195.

[55] Blaney, T. G., Edwards, G. J., Jolliffe, B. W., KNiGHT, D. J. E., Woods, P. T., J. Phys. D : Appl. Phys. 9 (1976) 1323-1330.

[56] Jennings, D. A., Petersen, F. R., Evenson, K. M., Appl. Phys. Lett. 26 (1975) 510-511.

[57] Abrams, R. L., Gandrud, W. B., Appl. Phys. Lett. 17 (1970) 150-152.

[58] Sullivan, T. E., lucas, A. A., Cutler, P. H., Appl. Phys. 14 (1977) 289-294.

[59] Sakuma, E., Evenson, K. M., IEEE J. Quant. Electr. QE-10 (1974) 599-603.

[60] Faris, S. M., Gustafson, T. K., Wiesner, J. C., IEEE Quant. Electr. QE-9 (1973) 737-745.

[61] Kwok, S. P., Haddad, G. I., Lonov, G., J. Appl. Phys. 42 (1971) 554-563

[62] Lucas, A. A., Moussiaux, A., Schmeits, M., Cutler, P. H., Comm. Phys. 2 (1977) 169-174.

[63] Comeron, A., Rapport stage DEA, Orsay, juin 1977

[64] Hartman, T. E., J. Appl. Phys., 33 (1962) 3427-3433.

[65] McDonald, D. G., Risley, A. S., CuPP, J. D., Evenson, K. M., Ashley, J. R., Appl. Phys. Lett. 20 (1972) 296-299.

[66] Blaney, T. G., Cross, N. R., Jones, R. G., Proc. 3nd Int Conf. Submillimetric Waves and their Applications, Univ. of Surrey, 29.3-1.4.1978, 258-259.

[67] Blaney, T. G., Knight, D. J. E., J. Phys. D. : Appl. Phys. 7 (1974) 1882-1886.

[68] Riedel, E., Z. Naturforsch. 19a (1964) 1634-1635.

[69] Wertamer, N. R., Phys. Rev. 147 (1966) 255-263.

[70] McDonald, D. G., Kose, V. E., Evenson, K. M., Wells, J. S., Cupp, J. D., Appl. Phys. Lett. 15 (1969) 121-122.

[71] Blaney, T. G., Cross, N. R., Knight, D. J. E., J. Phys. D Appl. Phys. 9 (1976) 2175-2180.

[72] McDonald, D. G., Petersen, F. R., Cupp, J. D., Danielson, B. L., Johnson, E. G., Appl. Phys. Lett. 24 (1974) 335-337.

[73] Jimenez, J., Rutman, J., Bull. d'Information du BNM, 7 (1976) 27-31.

[74] Vernet, G., Henaux, J. C., Adde, R., IEEE Trans. Microw. Theory Techn. MTT-25 (1977) 473-476.

[75] Taur, Y., KerR, A. R., Appl. Phys. Lett. 32 (1978) 775.

[76] Cette étude est réalisée en collaboration avec le groupe de ADDE, R. à l'Institut d'Electronique Fondamentale, Orsay.

[77] Risley, A. S., Proc. 31th An. Symposium Frequency Control, ed. Electronic Industries Assoc. Washington, DC, 1977, 583-589. 
[78] Stein, S. R., Risley, A. S., «Progress in far-infrared frequency synthesis ", CPEM'78 Conf. Ottawa, Canada, 26-29 juin 1978.

[79] Daiku, Y., Mizuno, K., Ono, S., Proc. 3rd Int. Conf. Submillimetric Waves and their Applications, Univ. of Surrey, 29.3-1.4. 1978, 255-256.

[80] Fetterman, H. R., Tannenwald, P. E., Clifton, B. J., Murphy, R. A., PARKer, C. D., Ibid., 241-242.

[81] Murphy, R. A., Bozler, C. O., Parker, C. D., Fetterman, H. R., Tannenwald, P. E., Clifton, B. J., Donnelly, J. P., Lindley, W. T., IEEE Trans. Microw. Theory Techn. MTT-25 (1977) 494-495.

[82] Small, J. G., Elchinger, G. M., Javan, A., Sanchez, A., Bachner, F. J., Smythe, D. L., Appl. Phys. Lett. 24 (1974) 275-279.

[83] Elchinger, G. M., Sanchez, A., Davis Jr. C. F., Javan, A., J. Appl. Phys. 47 (1976) 591-593.

[84] Gustafson, T. K., Schmidt, R. V., Peruca, J. R., Appl. Phys. Lett. 24 (1974) 620-622.
[85] Heiblum, M., Wang, S., Whinnery, J. R., Gustafson, T. K., IEEE J. Quant. Electr. QE-14 (1978) 159-169.

[86] Wells, J. S., IEEE Trans. Instr. Measur. IM-22 (1973) 113118.

[87] Godone, A., Weiss, C. O., Kramer, G., IEEE J. Quant. Electr. QE-14 (1978) 339-342.

[88] Jimenez, J. J., Plainchamp, P., Comeron, A., Clairon, A., "Submillimetric receivers : local oscillators and mixers" AGARD Symp. on Millimetric and Submillimetric Wave Propagation and Circuits, Munchen, RFA, 4-8 sept. 1978, papier 3.5.

[89] Plainchamp, P., Thèse de Doctorat $3^{\mathrm{e}}$ cycle, Paris, décembre 1978.

[90] Jimenez, J. J., " Microwave to visible frequency synthesis ", URSI Symp. Time and Frequency, Helsinki, Finlande, 31.7-8.8.1978 (à paraître dans Radio Science, 1979).

[91] Weiss, C. O., Communication privée, mai 1978.

[92] Petersen, F. R., Evenson, K. M., Jennings, D. A., Jimenez, J. J., Travail non publié, 1976. 